\title{
Weighted Estimates for Toeplitz Operators Related to Pseudodifferential Operators
}

\author{
Yan Lin, Zongguang Liu, Chengdan Xu, and Zichu Ren \\ School of Sciences, China University of Mining and Technology, Beijing 100083, China \\ Correspondence should be addressed to Yan Lin; linyan@cumtb.edu.cn \\ Received 7 January 2016; Accepted 10 February 2016 \\ Academic Editor: Dashan Fan
}

Copyright (c) 2016 Yan Lin et al. This is an open access article distributed under the Creative Commons Attribution License, which permits unrestricted use, distribution, and reproduction in any medium, provided the original work is properly cited.

\begin{abstract}
The authors establish the weighted $L^{p}$ estimates for a class of pseudodifferential operators for both cases $1<p<\infty$ and $p=1$, where the weight class is bigger than the classical Muckenhoupt's weight class. Moreover, the weighted estimates for the Toeplitz operators related to pseudodifferential operators are also obtained. As their special cases, the corresponding results for the commutators of pseudodifferential operators can be deduced.
\end{abstract}

\section{Introduction}

The pseudodifferential operator, appearing in 1960 s, is formalized in Kohn and Nirenberg [1] and Hörmander [2]. With the development of mathematic theory, the pseudodifferential operator has been a popular mathematic tool, which can be extensively applied in the research of various fields of mathematics, especially in dealing with some basic problems in the PDE.

The classical pseudodifferential operators are defined by Hörmander symbols. Following [3], let $m, \rho$, and $\delta$ be real numbers, and let $\sigma(x, \xi)$ be a smooth function, defined on $R^{n} \times R^{n}$ such that, for all multi-indices $\alpha$ and $\beta$, the following estimate

$$
\left|\partial_{x}^{\alpha} \partial_{\xi}^{\beta} \sigma(x, \xi)\right| \leq C_{\alpha, \beta}(1+|\xi|)^{m-\rho|\beta|+\delta|\alpha|}
$$

holds, where $C_{\alpha, \beta}>0$ is independent of $x$ and $\xi$; then, $\sigma(x, \xi)$ is called a symbol in the class $S_{\rho, \delta}^{m}$. A symbol in $S_{\rho, \delta}^{-\infty}$ is one which satisfies the above estimates for any real number $m$.

It can be deduced that $S_{\rho, \delta}^{m_{1}} \subset S_{\rho, \delta}^{m_{2}}$ if $m_{1} \leq m_{2}, S_{\rho_{2}, \delta}^{m} \subset S_{\rho_{1}, \delta}^{m}$ if $\rho_{1} \leq \rho_{2}$, and $S_{\rho, \delta_{1}}^{m} \subset S_{\rho, \delta_{2}}^{m}$ if $\delta_{1} \leq \delta_{2}$.

Definition 1. The operator $T$ given by

$$
T f(x)=\int_{R^{n}} \sigma(x, \xi) e^{2 \pi i x \cdot \xi} \hat{f}(\xi) d \xi
$$

is called a pseudodifferential operator with symbol $\sigma(x, \xi) \epsilon$ $S_{\rho, \delta}^{m}$, where $f$ is a Schwartz function (denote simply by $f \in \mathcal{S}$ ) and $\hat{f}$ denotes the Fourier transform of $f$.

As usual, $L_{\rho, \delta}^{m}$ will denote the class of pseudodifferential operators with symbols in $S_{\rho, \delta}^{m}$, and $L_{\rho, \delta}^{-\infty}$ will denote the class of pseudodifferential operators with symbols in $S_{\rho, \delta}^{-\infty}$.

The boundedness of different kinds of pseudodifferential operators on Lebesgue spaces and weighted Lebesgue spaces has been studied by many authorities. Laptev [4] proved that any pseudodifferential operator in $L_{1,0}^{0}$ is a standard Calderón-Zygmund operator, so the boundedness on $L^{p}$ is obvious. Hörmander [3] and Fefferman [5] pointed out that the pseudodifferential operators in $L_{\rho, \delta}^{m}$ are bounded on $L^{p}$ if and only if $m \leq n(\rho-1)|1 / p-1 / 2|$, where $0 \leq \delta \leq \rho \leq 1$, $\delta<1$, and $1<p<\infty$. Fefferman [5] also obtained the endpoint estimates for the pseudodifferential operators in $L_{\rho, \delta}^{m}$. Alvarez and Hounie [6] obtained the boundedness of the pseudodifferential operators in $L_{\rho, \delta}^{m}$ on $L^{p}$, where $0<\rho \leq 1,0 \leq \delta<1,1<p<\infty$, and $m \leq$ $n(\rho-1)|1 / p-1 / 2|+\min \{0, n(\rho-\delta) / 2\}$, in which condition $\delta \leq \rho$ is not necessary (also see [7, 8]). Lin and Lu [9] generalized the results in [10] and established the relationship between Lebesgue boundedness and Sobolev boundedness of pseudodifferential operators in related symbol classes. 
Miller [11] verified the boundedness of pseudodifferential operators in $L_{1,0}^{0}$ on weighted $L^{p}(1<p<\infty)$ spaces whenever the weight function belongs to Muckenhoupt's class $A_{p}\left(R^{n}\right)$. Tang [12] improved the result of Miller. Following [12], the boundedness of pseudodifferential operators in $L_{1,0}^{0}$ on weighted $L^{p}(1<p<\infty)$ spaces was verified whenever the weight function belongs to $A_{p}(\varphi)$, where for some $\alpha_{0}>0$, $\varphi(t)=(1+t)^{\alpha_{0}}, t \geq 0$, and the new class $A_{p}(\varphi)$ of weight functions is defined as follows.

Definition 2. A nonnegative measurable function $\omega \in$ $L_{\text {loc }}\left(R^{n}\right)$ is said to be in $A_{p}(\varphi)$ for $1<p<\infty$, if for every cube $Q$ in $R^{n}$ there exists a positive constant $C$ independent of $Q$ such that

$$
\left(\frac{1}{\varphi(|Q|)|Q|} \int_{Q} \omega(y) d y\right)\left(\frac{1}{\varphi(|Q|)|Q|} \int_{Q} \omega^{-1 /(p-1)}(y) d y\right)^{p-1}
$$

$\leq C$.

When $p=1$, a nonnegative measurable function $\omega$ is said to belong to $A_{1}(\varphi)$, if there exists a constant $C>0$ such that, for any cube $Q$,

$$
M_{\varphi}(\omega)(x) \leq C \omega(x), \quad \text { a.e. } x \in R^{n} .
$$

Here and in what follows, $1 / p+1 / p^{\prime}=1$, and

$$
M_{\varphi} f(x)=\sup _{Q \ni x} \frac{1}{\varphi(|Q|)|Q|} \int_{Q}|f(y)| d y .
$$

Denote $A_{\infty}(\varphi)=\bigcup_{1 \leq p<\infty} A_{p}(\varphi)$. Since $\varphi(|Q|) \geq 1$, $A_{p}\left(R^{n}\right) \subset A_{p}(\varphi)$ for $1 \leq p<\infty$, where $A_{p}\left(R^{n}\right)$ denote the classical Muckenhoupt weight class; see [13]. It is acknowledged that if $\omega \in A_{\infty}\left(R^{n}\right)=\bigcup_{p \geq 1} A_{p}\left(R^{n}\right)$, then $\omega(x) d x$ is a doubling measure. It follows from the definition and the properties of $A_{p}(\varphi)$ that if $\omega \in A_{p}(\varphi)$, then $\omega(x) d x$ may be not a doubling measure. In fact, let $0 \leq \gamma<n \alpha_{0}$; it is easy to check that $\omega(x)=(1+|x|)^{-(n+\gamma)}$ is not in $A_{\infty}\left(R^{n}\right)$ and $\omega(x) d x$ is not a doubling measure, but $\omega(x)=(1+|x|)^{-(n+\gamma)} \epsilon$ $A_{1}(\varphi) \subset A_{\infty}(\varphi)$. This fact implies that $A_{p}\left(R^{n}\right) \subsetneq A_{p}(\varphi), 1 \leq$ $p<\infty$.

Inspired by Tang [12], a natural question is whether the pseudodifferential operator in $L_{1, \delta}^{0}(0<\delta<1)$ is also bounded on weighted $L^{p}$ spaces with the weight function in $A_{p}(\varphi)$.

In 1976, Coifman et al. [14] introduced a class of nonconvolution operators, which is said to be the commutator generated by a singular integral operator $T$ and a function $b$ :

$$
[b, T] f(x)=b(x) T f(x)-T(b f)(x) .
$$

Auscher and Taylor [15] and Taylor [16] investigated the boundedness of the commutator $[b, T]$ generated by the pseudodifferential operators $T$ in $L_{\rho, \delta}^{m}(\rho=1)$ and a suitable function $b$ on $L^{p}, 1 \leq p<\infty$. Alvarez and Hounie [6] introduced $L^{p}(1 \leq p<\infty)$ boundedness results for the commutator $[b, T]$ generated by the pseudodifferential operators $T$ in $L_{\rho, \delta}^{m}$ and a function $b \in \mathrm{BMO}$, wherever $0<\rho \leq(1 / 2)(1-2 n \lambda /(n+2))$ or $\rho=1,0 \leq \delta<1$, $\lambda=\max \{0,(\delta-\rho) / 2\}, m \leq-n(1-\rho)-\mu$, and $2 \mu=$ $1+n(\rho+\lambda)-\sqrt{[1+n(\rho+\lambda)]^{2}-4 n \lambda}$. Chanillo [7] obtained the boundedness on $L^{2}$ for the commutator $[b, T]$ generated by the pseudodifferential operators $T$ in $L_{-\varepsilon, \delta}^{m}$ and a function $b \in \mathrm{BMO}$, wherever $\varepsilon>0$ and $0 \leq \delta<\rho<1$. Lin [17] partly improved the results in $[6,7]$ and established the boundedness on $L^{p}$ for the commutator $[b, T]$ generated by the pseudodifferential operators $T$ in $L_{\rho, \delta}^{m}$ and a function $b \in$ $\mathrm{BMO}$, where $0<\rho \leq(1 / 2)(1-2 n \lambda /(n+2)), 0 \leq \delta<1, \lambda=$ $\max \{0,(\delta-\rho) / 2\}, 1<p<2$, and $m<-n[(1-\rho)(1 / p-1 / 2)+\lambda]$ or $0 \leq \delta<\rho \leq 1 / 2,1<p<\infty$, and $m \leq n(\rho-1)|1 / p-1 / 2|$. Michalowski et al. [18] constructed the $L^{p}$ boundedness for the commutator $[b, T]$ generated by the pseudodifferential operators $T$ in $L_{\rho, \delta}^{m}$ and a function $b \in \mathrm{BMO}$, where $0<\rho \leq 1$, $0 \leq \delta<1,1<p<\infty$, and $m \leq n(\rho-1)|1 / p-1 / 2|+$ $\min \{0, n(\rho-\delta) / 2\}$. The boundedness of commutators $[b, T]$ generated by pseudodifferential operators $T$ in $L_{1,0}^{0}$ and BMO functions on weighted $L^{p}(1<p<\infty)$ spaces was obtained with the weight functions in $A_{p}(\varphi)$ in [12].

The boundedness of the Toeplitz operator was introduced by Krantz and Li [19] in 2001. And the commutator of the Calderón-Zygmund operators can be regarded as a special case of the Toeplitz operator $T_{b}=\sum_{j=1}^{m} T_{j, 1} M_{b} T_{j, 2}$, where $T_{j, 1}$ and $T_{j, 2}$ are the Calderón-Zygmund operators or $\pm I$ ( $I$ is the identity operator) and $M_{b} f(x)=b(x) f(x)$ is a multiplication operator. When $b \in \mathrm{BMO}, \mathrm{Krantz}$ and $\mathrm{Li}$ [19] discussed the $L^{p}$ boundedness of $T_{b}$ on the homogeneous space.

In this paper, we also concentrated on the weighted Lebesgue boundedness of Toeplitz operators $T_{b}$ related to pseudodifferential operators and BMO functions. Actually, in this situation, $T_{j, 1}$ and $T_{j, 2}$ are pseudodifferential operators in $L_{1, \delta}^{0}(0<\delta<1)$ or $\pm I$, and $b \in$ BMO. Moreover, as a special case, we can obtain the corresponding results of the commutators generated by pseudodifferential operators and BMO functions on weighted Lebesgue spaces.

Before stating our main results, we firstly recall some notations and definitions as follows.

Given $B=B(x, r)$ and $\lambda>0, \lambda B$ denote the $\lambda$-dilate ball, which is the ball with the same center $x$ and radius $\lambda r$. Similarly, $Q(x, r)$ denote the cube centered at $x$ with the side length $r$, where the sides of the cubes are parallel to the coordinate axes. Given a Lebesgue measurable set $E$ and a weight $\omega,|E|$ will denote the Lebesgue measure of $E$ and $\omega(E)=$ $\int_{E} \omega(x) d x .\|f\|_{L^{p}(\omega)}$ will denote $\left(\int_{R^{n}}|f(y)|^{p} \omega(y) d y\right)^{1 / p}$ for $1 \leq p<\infty$. We denote $C$ by the constants that are independent of the main parameters involved but whose value may differ from line to line. For a measurable set $E$, denote by $\chi_{E}$ the characteristic function of $E$. By $A \sim B$, we mean that there exists a constant $C>1$ such that $1 / C \leq$ $A / B \leq C$.

Definition 3. The Hardy-Littlewood maximal operator $M$ is defined by

$$
M f(x)=\sup _{Q \ni x} \frac{1}{|Q|} \int_{Q}|f(y)| d y .
$$


Definition 4. The maximal operator $M_{\varphi, \eta}$ for $0<\eta<\infty$ is defined by

$$
M_{\varphi, \eta} f(x)=\sup _{Q \ni x} \frac{1}{\varphi(|Q|)^{\eta}|Q|} \int_{Q}|f(y)| d y .
$$

Definition 5. The dyadic maximal operator $M_{\varphi, \eta}^{\Delta}$ for $0<\eta<$ $\infty$ is defined by

$$
M_{\varphi, \eta}^{\Delta} f(x)=\sup _{Q \ni x} \frac{1}{\varphi(|Q|)^{\eta}|Q|} \int_{Q}|f(y)| d y,
$$

where $Q$ denotes any dyadic cube containing $x$ in $R^{n}$.

A variant of dyadic maximal operator $M_{s, \varphi, \eta}^{\Delta}$ for $0<\eta<$ $\infty$ and $0<s<\infty$ is defined by

$$
M_{s, \varphi, \eta}^{\Delta} f(x)=M_{\varphi, \eta}^{\Delta}\left(|f|^{s}\right)^{1 / s}(x) .
$$

Definition 6. The dyadic sharp maximal operator $M_{\varphi, \eta}^{\sharp, \Delta}$ for $0<\eta<\infty$ is defined by

$$
\begin{aligned}
M_{\varphi, \eta}^{\sharp, \Delta} f(x)= & \sup _{Q \ni x, r<1} \frac{1}{|Q|} \int_{Q}\left|f(y)-f_{Q}\right| d y \\
& +\sup _{Q \ni x, r \geq 1} \frac{1}{\varphi(|Q|)^{\eta}|Q|} \int_{Q}|f(y)| d y \\
\sim & \sup _{Q \ni x, r<1} \inf _{c} \frac{1}{|Q|} \int_{Q}|f(y)-c| d y \\
& +\sup _{Q \ni x, r \geq 1} \frac{1}{\varphi(|Q|)^{\eta}|Q|} \int_{Q}|f(y)| d y,
\end{aligned}
$$

where $Q$ denotes any dyadic cube containing $x$ in $R^{n}$ and $f_{Q}=$ $(1 /|Q|) \int_{Q}|f(y)| d y$.

A variant of dyadic sharp maximal operator $M_{s, \varphi, \eta}^{\sharp, \Delta}$ for $0<$ $\eta<\infty$ and $0<s<\infty$ is defined by

$$
M_{s, \varphi, \eta}^{\sharp, \Delta} f(x)=M_{\varphi, \eta}^{\sharp, \Delta}\left(|f|^{s}\right)^{1 / s}(x) .
$$

Definition 7. A function $B(t):[0, \infty) \rightarrow[0, \infty)$ is called a Young function if it is continuous, convex, and increasing and satisfies $B(0)=0$ and $B(t) \rightarrow \infty$ as $t \rightarrow \infty$. If $B$ is a Young function, we define the $B$-average of a function $f$ over a cube $Q$ by means of the following Luxemburg norm:

$$
\|f\|_{B, Q}=\inf \left\{\lambda>0: \frac{1}{|Q|} \int_{Q} B\left(\frac{|f(y)|}{\lambda}\right) d y \leq 1\right\} .
$$

The generalized Hölder inequality

$$
\frac{1}{|Q|} \int_{Q}|f g| \leq\|f\|_{B, Q}\|G\|_{\bar{B}, Q}
$$

holds, where $\bar{B}$ is the complementary Young function associated with $B$. And we define the corresponding maximal function

$$
\begin{aligned}
M_{B} f(x) & =\sup _{\mathrm{Q} \ni x}\|f\|_{B, Q}, \\
M_{B, \varphi, \eta} f(x) & =\sup _{Q \ni x} \varphi(|Q|)^{-\eta}\|f\|_{B, Q} .
\end{aligned}
$$

The example we are going to use in Section 3 is $B(t)=$ $t\left(1+\log ^{+} t\right)$ with the maximal function denoted by $M_{L \log L}$. The complementary Young function is given by $\bar{B}(t) \approx e^{t}$ with the maximal function denoted by $M_{\exp L}$.

Now we define a function, which will be used in Section 3. It is a negative, Radial, and $C^{\infty}$ function of compact support, defined in the $\xi$-space $R^{n}$, with the properties that $\nu(\xi)=1$ for $|\xi| \leq 1$ and $\nu(\xi)=0$ for $|\xi| \geq 2$. Together with $\nu$, we define another function $\phi$, by $\phi(\xi)=\nu(\xi)-\nu(2 \xi)$. Then, we have the following partitions of unity of the $\xi$-space:

$$
1=\nu(\xi)+\sum_{j=1}^{\infty} \phi\left(2^{-j} \xi\right), \quad \forall \xi \in R^{n}
$$

Let $\theta$ and $\psi$, respectively, denote the reverse Fourier transform of $\nu$ and $\phi$; that is,

$$
\begin{gathered}
\widehat{\theta}(\xi)=\nu(\xi), \\
\widehat{\psi}(\xi)=\phi(\xi) .
\end{gathered}
$$

Write $\theta_{t}(x)=t^{-n} \theta(x / t)$ and $\psi_{t}(x)=t^{-n} \psi(x / t)$; then, $\widehat{\theta}_{2^{-j}}(\xi)=\nu\left(2^{-j} \xi\right)$ and $\widehat{\psi}_{2^{-j}}(\xi)=\phi\left(2^{-j} \xi\right)$.

The organization of the paper is as follows. The main results are showed in Section 2. Lemmas that we need to prove our main results are accounted for in Section 3. The proof of the main results is in Section 4.

\section{Main Results}

Now we state our main results. Firstly, we establish the weighted boundedness for the pseudodifferential operators in $L_{1, \delta}^{0}, 0<\delta<1$.

Theorem 8. Suppose $T \in L_{1, \delta}^{0}, 0<\delta<1$. If $\omega \in A_{p}(\varphi)$, $1<p<\infty$, then $T$ is bounded on $L^{p}(\omega)$.

Theorem 9. Suppose $T \in L_{1, \delta}^{0}, 0<\delta<1$. If $\omega \in A_{1}(\varphi)$, then there exists a constant $C>0$ such that, for any $\lambda>0$,

$$
\omega\left(\left\{x \in R^{n}:|T f(x)|>\lambda\right\}\right) \leq \frac{C}{\lambda} \int_{R^{n}}|f(x)| \omega(x) d x .
$$

Then, we obtain the weighted estimates for the Toeplitz operators $T_{b}$ related to pseudodifferential operators and $\mathrm{BMO}$ functions.

Theorem 10. Suppose $m \in \mathbb{N}$ and $T_{j, k} \in L_{1, \delta}^{0}, 0<\delta<1$, or $T_{j, k}= \pm I$, where $k=1,2, j=1,2, \ldots, m$. Let $T_{1}(f)=0$ when $f \in C_{0}^{\infty}\left(R^{n}\right)$. If $b \in B M O$ and $\omega \in A_{p}(\varphi), 1<p<\infty$, then $T_{b}$ is bounded on $L^{p}(\omega)$.

Since the commutator can be seen as a special case of the Toeplitz operator, we can get the following result as a corollary of Theorem 10.

Theorem 11. Suppose $T \in L_{1, \delta}^{0}, 0<\delta<1$. If $b \in B M O$ and $\omega \in A_{p}(\varphi), 1<p<\infty$, then $[b, T]$ is bounded on $L^{p}(\omega)$. 
At last, we can also establish the endpoint estimate for the commutator.

Theorem 12. Suppose $T \in L_{1, \delta}^{0}, 0<\delta<1$. If $b \in B M O$ and $\omega \in A_{1}(\varphi)$, then there exists a constant $C>0$ such that, for any $\lambda>0$,

$$
\begin{aligned}
& \omega\left(\left\{x \in R^{n}:|[b, T] f(x)|>\lambda\right\}\right) \\
& \quad \leq C \int_{R^{n}} \frac{|f(x)|}{\lambda}\left(1+\log ^{+}\left(\frac{|f(x)|}{\lambda}\right)\right) \omega(x) d x .
\end{aligned}
$$

\section{Necessary Lemmas}

Before giving the proof of our main results, we need some necessary lemmas. In this section, we are inspired by some ideas from $[10,12,14,18,20-22]$.

Lemma 13 (see [12]). Let $\omega \in A_{p_{1}}(\varphi)$ and $1 \leq p_{1}<\infty$. If $p_{1}<p<\infty$, then

$$
\int_{R^{n}}\left|M_{\varphi} f(x)\right|^{p} \omega(x) d x \leq C_{p} \int_{R^{n}}|f(x)|^{p} \omega(x) d x .
$$

Furthermore, if $1 \leq p<\infty$, then $\omega \in A_{p}(\varphi)$ if and only if

$$
\begin{gathered}
\omega\left(\left\{x \in R^{n}: M_{\varphi} f(x)>\lambda\right\}\right) \\
\leq \frac{C_{p}}{\lambda^{p}} \int_{R^{n}}|f(x)|^{p} \omega(x) d x .
\end{gathered}
$$

Lemma 14. Suppose $A \in L_{1, \delta}^{-\infty}, 0<\delta<1$ and $0<\eta<\infty$, then there exists a constant $C>0$ such that, for any $x_{0} \in R^{n}$ and $f \in \mathcal{S}$,

$$
M_{\varphi, \eta}^{\sharp, \Delta}(A f)\left(x_{0}\right) \leq C M_{\varphi, \eta} f\left(x_{0}\right) .
$$

Proof. Let $a(x, \xi)$ be the symbol of $A$; then, for all real numbers $m$ and any multi-indices $\alpha$ and $\beta$, there exists a constant $C_{m, \alpha, \beta}>0$ such that

$$
\left|\partial_{x}^{\alpha} \partial_{\xi}^{\beta} a(x, \xi)\right| \leq C_{m, \alpha, \beta}(1+|\xi|)^{m-|\beta|+\delta|\alpha|} .
$$

Write

$$
\begin{aligned}
A f(x) & =\int_{R^{n}} a(x, \xi) e^{2 \pi i x \cdot \xi} \hat{f}(\xi) d \xi \\
& =\int_{R^{n}} K(x, y) f(y) d y,
\end{aligned}
$$

where

$$
K(x, y)=\int_{R^{n}} a(x, \xi) e^{2 \pi i(x-y) \cdot \xi} d \xi
$$

First of all, we will prove that, for any $k>0$, there is a constant $C_{k}>0$, such that, for all $x, y \in R^{n}$,

$$
|K(x, y)| \leq C_{k}(1+|x-y|)^{-k}
$$

In fact,

$$
\begin{aligned}
K(x, y)= & \int_{|\xi| \leq 1+|x-y|} a(x, \xi) e^{2 \pi i(x-y) \cdot \xi} d \xi \\
& +\int_{|\xi|>1+|x-y|} a(x, \xi) e^{2 \pi i(x-y) \cdot \xi} d \xi \\
:= & K_{1}+K_{2} .
\end{aligned}
$$

Now we estimate $K_{1}$.

Case 1. If $|x-y| \leq 1$, then by taking $m \leq 0$ in (23), we have

$$
\left|K_{1}\right| \leq C \leq C_{k}(1+|x-y|)^{-k} .
$$

Case 2. If $|x-y|>1$, then $1+|x-y| \sim|x-y|$. Through repeating integration by parts, we can obtain

$$
\left|K_{1}\right| \leq \sum_{l=1}^{k_{1}} I_{l}+I
$$

where $k_{1}=[k]+1 ;[k]$ expresses the biggest integer no more than $k$.

Take $m=2 k_{1}-n-k$ in $(23)$; then, $(1+|\xi|)^{m-k_{1}} \leq|\xi|^{m-k_{1}}$ and

$$
\begin{aligned}
I & \leq \frac{C}{|x-y|^{k_{1}}} \int_{|\xi| \leq 1+|x-y|}(1+|\xi|)^{m-k_{1}} d \xi \\
& \leq \frac{C}{|x-y|^{k_{1}}} \int_{|\xi| \leq 1+|x-y|}|\xi|^{m-k_{1}} d \xi \\
& \leq C_{k}(1+|x-y|)^{-k} .
\end{aligned}
$$

Take $m=2 l-1-k$ in (23); then,

$$
\begin{aligned}
I_{l} & \leq \frac{C}{|x-y|^{l}}(1+|x-y|)^{m-(l-1)} \\
& \leq C_{k}(1+|x-y|)^{-k}
\end{aligned}
$$

Thus, $\left|K_{1}\right| \leq C_{k}(1+|x-y|)^{-k}$

Next we estimate $K_{2}$. Take $m=-n-k$ in (23); then,

$$
\left|K_{2}\right| \leq C_{k} \int_{|\xi|>1+|x-y|}|\xi|^{m} d \xi \leq C_{k}(1+|x-y|)^{-k}
$$

From the above, we get the inequality we desired. Then, for any $k>0$,

$$
|A f(x)| \leq C_{k} \int_{R^{n}} \frac{|f(y)|}{(1+|x-y|)^{k}} d y .
$$

Thus, by taking $k>n$ in (33), we have

$$
\|A f\|_{L^{1}} \leq C\|f\|_{L^{1}} .
$$


For any dyadic cube $Q=Q\left(x_{1}, r\right) \ni x_{0}$, let $\bar{Q}=Q\left(x_{1}\right.$, $4 \sqrt{n} r)$. We write

$$
f=f \chi_{\bar{Q}}+f \chi_{(\bar{Q})^{c}}:=f_{1}+f_{2} .
$$

Take $k>n+n \alpha_{0} \eta$ in (33); then,

$$
\begin{aligned}
& \frac{1}{\varphi(|Q|)^{\eta}|Q|} \int_{Q}|A f(x)| d x \\
& \leq \frac{1}{\varphi(|Q|)^{\eta}|Q|} \int_{Q}\left|A f_{1}(x)\right| d x \\
& +\frac{1}{\varphi(|Q|)^{\eta}|Q|} \int_{Q}\left|A f_{2}(x)\right| d x \\
& \leq \frac{C}{\varphi(|Q|)^{n}|Q|} \int_{R^{n}}\left|f_{1}(x)\right| d x \\
& +\frac{C_{k}}{\varphi(|Q|)^{\eta}|Q|} \int_{Q} \int_{R^{n}} \frac{\left|f_{2}(y)\right|}{(1+|x-y|)^{k}} d y d x \\
& \leq \frac{C}{\varphi(|Q|)^{\eta}|Q|} \frac{\varphi(|\bar{Q}|)^{\eta}|\bar{Q}|}{\varphi(|\bar{Q}|)^{\eta}|\bar{Q}|} \int_{\bar{Q}}|f(x)| d x \\
& +\frac{C_{k}}{\varphi(|Q|)^{\eta}} \int_{\left|y-x_{0}\right|>\sqrt{n} r} \frac{|f(y)|}{\left(1+\left|x_{0}-y\right|\right)^{k}} d y \\
& \leq C M_{\varphi, \eta} f\left(x_{0}\right) \\
& +\frac{C_{k}}{\varphi(|Q|)^{\eta}} \int_{\sqrt{n} r<\left|y-x_{0}\right|<1} \frac{|f(y)|}{\left(1+\left|x_{0}-y\right|\right)^{k}} d y \\
& +\frac{C_{k}}{\varphi(|Q|)^{\eta}} \int_{\left|y-x_{0}\right| \geq 1} \frac{|f(y)|}{\left(1+\left|x_{0}-y\right|\right)^{k}} d y \\
& \leq C M_{\varphi, \eta} f\left(x_{0}\right)+C_{k} \int_{\mathrm{Q}_{1}\left(x_{0}, 2\right)}|f(y)| d y \\
& +C_{k} \sum_{j=0}^{\infty} \int_{2^{j} \leq\left|y-x_{0}\right|<2^{j+1}} \frac{|f(y)|}{\left|x_{0}-y\right|^{k}} d y \\
& \leq C M_{\varphi, \eta} f\left(x_{0}\right) \\
& +C_{k} \frac{\varphi\left(\left|Q_{1}\right|\right)^{\eta}\left|Q_{1}\right|}{\varphi\left(\left|Q_{1}\right|\right)^{\eta}\left|Q_{1}\right|} \int_{Q_{1}\left(x_{0}, 2\right)}|f(y)| d y \\
& +C_{k} \sum_{j=0}^{\infty} \frac{\varphi\left(\left|Q_{2}\right|\right)^{\eta}\left|Q_{2}\right|}{\varphi\left(\left|Q_{2}\right|\right)^{\eta}\left|Q_{2}\right|} \int_{Q_{2}\left(x_{0}, 2^{j+2}\right)} \frac{|f(y)|}{2^{j k}} d y \\
& \leq C M_{\varphi, \eta} f\left(x_{0}\right)+C_{k} M_{\varphi, \eta} f\left(x_{0}\right) \\
& +C_{k} \sum_{j=0}^{\infty} 2^{j\left(-k+n \alpha_{0} \eta+n\right)} M_{\varphi, \eta} f\left(x_{0}\right) \\
& \leq C M_{\varphi, \eta} f\left(x_{0}\right) \text {. }
\end{aligned}
$$

Taking the supremum of all dyadic cubes $Q$ containing $x_{0}$, we can achieve the inequality

$$
M_{\varphi, \eta}^{\Delta}(A f)\left(x_{0}\right) \leq C M_{\varphi, \eta} f\left(x_{0}\right) .
$$

Since

$$
\begin{aligned}
& M_{\varphi, \eta}^{\sharp, \Delta}(A f)\left(x_{0}\right) \\
& =\sup _{\mathrm{Q} \ni x_{0}, r<1} \frac{1}{|Q|} \int_{Q}\left|A f(x)-(A f)_{Q}\right| d x \\
& \quad+\sup _{Q \ni x_{0}, r \geq 1} \frac{1}{\varphi(|Q|)^{\eta}|Q|} \int_{Q}|A f(x)| d x,
\end{aligned}
$$

where $Q$ denotes any dyadic cube containing $x_{0}$ in $R^{n}$, then

$$
\begin{aligned}
M_{\varphi, \eta}^{\sharp \Delta}(A f)\left(x_{0}\right) & \\
\leq & 2^{\alpha_{0} \eta} \sup _{\mathrm{Q} \ni x_{0}, r<1} \frac{2}{\varphi(|Q|)^{\eta}|Q|} \int_{Q}|A f(x)| d x \\
& \quad+M_{\varphi, \eta}^{\Delta}(A f)\left(x_{0}\right) \leq C M_{\varphi, \eta}^{\Delta}(A f)\left(x_{0}\right) \\
\leq & C M_{\varphi, \eta} f\left(x_{0}\right),
\end{aligned}
$$

which completes the proof of this lemma.

Inspired by the results in $[23,24]$, we have the following lemma.

Lemma 15. Suppose $q(x, \xi) \in S_{1, \delta}^{m}, 0<\delta<1, m \in R, \phi \in$ $C_{0}^{\infty}\left(R^{n}\right)$, and $\phi$ is supported on $\{\xi: 1 / 2 \leq|\xi| \leq 2\}$. If $N \geq 0$, then there exists a constant $C_{N}>0$ such that the inequality

$$
|y|^{N}\left|\int_{R^{n}} q(x, \xi) \phi\left(2^{-j} \xi\right) e^{2 \pi i y \cdot \xi} d \xi\right| \leq C_{N} 2^{j(n+m-N)}
$$

holds for all $x$ and $y$ in $R^{n}$ and every integer $j \geq 1$.

Proof.

Case 1. $N \geq 0$ and $N$ is an integer.

Let $y=\left(y_{1}, y_{2}, \ldots, y_{n}\right)$ and $|y|_{\infty}=\max \left\{\left|y_{i}\right|, i=\right.$ $1,2, \ldots, n\}$; then, $|y| \leq \sqrt{n}|y|_{\infty}$. Take $i$ such that $|y|_{\infty}=\left|y_{i}\right|$; then,

$$
\begin{aligned}
& |y|^{N}\left|\int_{R^{n}} q(x, \xi) \phi\left(2^{-j} \xi\right) e^{2 \pi i y \cdot \xi} d \xi\right| \\
& \quad \leq C_{N}|y|_{\infty}^{N}\left|\int_{R^{n}} q(x, \xi) \phi\left(2^{-j} \xi\right) e^{2 \pi i y \cdot \xi} d \xi\right| \\
& \quad \leq C_{N}\left|\int_{R^{n}} q(x, \xi) \phi\left(2^{-j} \xi\right)\left[\left(\frac{\partial}{\partial \xi_{i}}\right)^{N} e^{2 \pi i y \cdot \xi}\right] d \xi\right| \\
& \quad=C_{N}\left|\int_{R^{n}}\left(\frac{\partial}{\partial \xi_{i}}\right)^{N}\left[q(x, \xi) \phi\left(2^{-j} \xi\right)\right] e^{2 \pi i y \cdot \xi} d \xi\right| \\
& \quad \leq C_{N} \int_{2^{j-1} \leq|\xi| \leq 2^{j+1}} 2^{j(m-N)} d \xi \leq C_{N} 2^{j(m+n-N)} .
\end{aligned}
$$


Case 2. $N \geq 0$ and $N$ is not an integer; then, there exists a nonnegative integer $i$ such that $i \leq N<i+1$.

Subcase 1. If $2^{j}|y|>1$, then $\left(2^{j}|y|\right)^{N} \leq\left(2^{j}|y|\right)^{i+1}$ :

$$
\begin{aligned}
& |y|^{N}\left|\int_{R^{n}} q(x, \xi) \phi\left(2^{-j} \xi\right) e^{2 \pi i y \cdot \xi} d \xi\right| \\
& \quad \leq\left(2^{j}|y|\right)^{i+1}\left|\int_{R^{n}} q(x, \xi) \phi\left(2^{-j} \xi\right) e^{2 \pi i y \cdot \xi} d \xi\right| 2^{-j N} \\
& \quad \leq C_{N} 2^{j(i+1-N)} 2^{j(n+m-i-1)}=C_{N} 2^{j(m+n-N)} .
\end{aligned}
$$

Subcase 2. If $2^{j}|y| \leq 1$, then $\left(2^{j}|y|\right)^{N} \leq\left(2^{j}|y|\right)^{i}$ :

$$
\begin{aligned}
& |y|^{N}\left|\int_{R^{n}} q(x, \xi) \phi\left(2^{-j} \xi\right) e^{2 \pi i y \cdot \xi} d \xi\right| \\
& \quad \leq\left(2^{j}|y|\right)^{i}\left|\int_{R^{n}} q(x, \xi) \phi\left(2^{-j} \xi\right) e^{2 \pi i y \cdot \xi} d \xi\right| 2^{-j N} \\
& \quad \leq C_{N} 2^{j(i-N)} 2^{j(n+m-i)}=C_{N} 2^{j(m+n-N)} .
\end{aligned}
$$

Thus, we complete the proof of the lemma.

Lemma 16. If $T \in L_{1, \delta}^{0}, 0<\delta<1$, then, for any $0<\eta<\infty$ and $0<s<1$, there exists a constant $C>0$ such that, for any $f \in \mathcal{S}$,

$$
M_{s, \varphi, \eta}^{\sharp, \Delta}(T f)\left(x_{0}\right) \leq C M_{\varphi, \eta}(f)\left(x_{0}\right) \text {, a.e. } x_{0} \in R^{n} .
$$

Proof. Let $a(x, \xi)$ be the symbol of $T$. Firstly, we suppose that $a(x, \xi)$ has compact $\xi$-support and prove the various constants that occur in the following arguments will not depend on the support of $a$ :

$$
\begin{aligned}
& M_{s, \varphi, \eta}^{\sharp, \Delta}(T f)\left(x_{0}\right) \\
& \leq C \sup _{Q \ni x_{0}, r<1}\left(\left.\inf _{c} \frac{1}{|Q|} \int_{Q}|| T f(x)\right|^{s}-c \mid d x\right)^{1 / s} \\
& \quad+C \sup _{Q \ni x_{0}, r \geq 1}\left(\frac{1}{\varphi(|Q|)^{\eta}|Q|} \int_{Q}|T f(x)|^{s} d x\right)^{1 / s} .
\end{aligned}
$$

For any dyadic cube $Q=Q\left(x_{1}, r\right) \ni x_{0}$, let $\bar{Q}=Q\left(x_{1}\right.$, $4 \sqrt{n} r)$. Decompose $f=f \chi_{\bar{Q}}+f \chi_{(\bar{Q})^{c}}:=f_{1}+f_{2}$.

Case 1. When $r<1$, take $c=\left|\left(T f_{2}\right)_{\mathrm{Q}}\right|^{s}$. Since $\left.|| a\right|^{s}-|b|^{s} \mid \leq$ $|a-b|^{s}$, then

$$
\begin{aligned}
\inf _{c}( & \left.\left.\frac{1}{|Q|} \int_{Q}|| T f(x)\right|^{s}-c \mid d x\right)^{1 / s} \\
\leq & \left(\frac{1}{|Q|} \int_{Q}\left|T f(x)-\left(T f_{2}\right)_{Q}\right|^{s} d x\right)^{1 / s} \\
\leq & C\left(\frac{1}{|Q|} \int_{Q}\left|T f_{1}(x)\right|^{s} d x\right)^{1 / s} \\
& +C\left(\frac{1}{|Q|} \int_{Q}\left|T f_{2}(x)-\left(T f_{2}\right)_{Q}\right|^{s} d x\right)^{1 / s} \\
:= & H_{1}+H_{2} .
\end{aligned}
$$

Firstly, we estimate $H_{1}$. According to [24], we know that $T$ is of weak type $(1,1)$. By Kolmogorov inequality, we get

$$
\begin{aligned}
H_{1} & \leq C\left(\frac{1}{|Q|}\right)^{1 / s}\left(|Q|^{1-s}\left\|f_{1}\right\|_{1}^{s}\right)^{1 / s} \\
& =C \frac{1}{|Q|} \int_{\bar{Q}}|f(x)| d x \\
& \leq C \frac{1}{\varphi(|\bar{Q}|)^{\eta}|\bar{Q}|} \int_{\bar{Q}}|f(x)| d x \leq C M_{\varphi, \eta} f\left(x_{0}\right) .
\end{aligned}
$$

Secondly, we estimate $\mathrm{H}_{2}$. Decompose the operator $\mathrm{T}$ into a sum of simpler operators. Since

$$
1=\nu(\xi)+\sum_{j=1}^{\infty} \phi\left(2^{-j} \xi\right), \quad \forall \xi \in R^{n},
$$

we can write

$$
\begin{aligned}
T f_{2}(x)= & \int_{R^{n}} \widehat{f}_{2}(\xi) a(x, \xi) \nu(\xi) e^{2 \pi i x \cdot \xi} d \xi \\
& +\sum_{j=1}^{\infty} \int_{R^{n}} \widehat{f}_{2}(\xi) a(x, \xi) \phi\left(2^{-j} \xi\right) e^{2 \pi i x \cdot \xi} d \xi \\
:= & A f_{2}(x)+\sum_{j=1}^{\infty} A_{j} f_{2}(x) .
\end{aligned}
$$

It follows from $a(x, \xi) \in S_{1, \delta}^{0}$ that $a(x, \xi) \nu(\xi) \in S_{1, \delta}^{m}$ for any $m \in R$; thus, $A \in L_{1, \delta}^{-\infty}$. By Lemma 14, we have

$$
\begin{aligned}
H_{2} \leq & \frac{C}{|Q|} \int_{Q}\left|T f_{2}(x)-\left(T f_{2}\right)_{Q}\right| d x \\
\leq & \frac{C}{|Q|} \int_{Q}\left|A f_{2}(x)-\left(A f_{2}\right)_{Q}\right| d x \\
& +\frac{C}{|Q|} \int_{Q}\left|\sum_{j=1}^{\infty} A_{j} f_{2}(x)-\sum_{j=1}^{\infty}\left(A_{j} f_{2}\right)_{\mathrm{Q}}\right| d x \\
\leq & C M_{\varphi, \eta}^{\sharp, \Delta}\left(A f_{2}\right)\left(x_{0}\right) \\
& +C \sum_{j=1}^{\infty} \frac{1}{|Q|} \int_{Q}\left|A_{j} f_{2}(x)-\left(A_{j} f_{2}\right)_{\mathrm{Q}}\right| d x \\
\leq & C M_{\varphi, \eta} f\left(x_{0}\right) \\
& +C \sum_{j=1}^{\infty} \frac{1}{|Q|} \int_{Q}\left|A_{j} f_{2}(x)-\left(A_{j} f_{2}\right)_{\mathrm{Q}}\right| d x .
\end{aligned}
$$

Write

$$
\begin{gathered}
\frac{1}{|Q|} \int_{Q}\left|A_{j} f_{2}(x)-\left(A_{j} f_{2}\right)_{\mathrm{Q}}\right| d x \leq \frac{1}{|Q|} \frac{1}{|Q|} \\
\cdot \int_{Q} \int_{Q} \int_{R^{n}}\left|f_{2}(y)\right| \mid \int_{R^{n}}\left[a(x, \xi) e^{2 \pi i(x-y) \cdot \xi}\right. \\
\left.-a(z, \xi) e^{2 \pi i(z-y) \cdot \xi}\right] \phi\left(2^{-j} \xi\right) d \xi \mid d y d z d x .
\end{gathered}
$$


Subcase 1. If $2^{j} r \geq 1$, then take $k_{0} \in Z$ such that $1 / 2<2^{k_{0}} r \leq$ 1 :

$$
\begin{aligned}
& \frac{1}{|Q|} \int_{Q}\left|A_{j} f_{2}(x)-\left(A_{j} f_{2}\right)_{\mathrm{Q}}\right| d x \leq \frac{2}{|Q|} \int_{\mathrm{Q}} \int_{R^{n}}\left|f_{2}(y)\right| \\
& \cdot\left|\int_{R^{n}} a(x, \xi) e^{2 \pi i(x-y) \cdot \xi} \phi\left(2^{-j} \xi\right) d \xi\right| d y d x \\
& \quad \leq \sum_{k=1}^{k_{0}} \frac{2}{|Q|} \int_{Q} \int_{2^{k} \sqrt{n} r \leq\left|y-x_{1}\right|<2^{k+1} \sqrt{n} r}|f(y)| \\
& \cdot\left|\int_{R^{n}} a(x, \xi) e^{2 \pi i(x-y) \cdot \xi} \phi\left(2^{-j} \xi\right) d \xi\right| d y d x \\
& \quad+\sum_{k=k_{0}+1}^{\infty} \frac{2}{|Q|} \int_{Q} \int_{2^{k} \sqrt{n} r \leq\left|y-x_{1}\right|<2^{k+1} \sqrt{n} r}|f(y)| \\
& \quad \cdot\left|\int_{R^{n}} a(x, \xi) e^{2 \pi i(x-y) \cdot \xi} \phi\left(2^{-j} \xi\right) d \xi\right| d y d x:=J_{1} \\
& \quad+J_{2} .
\end{aligned}
$$

Now we estimate $J_{1}$. Let $Q_{k}=Q\left(x_{1}, 2^{k+2} \sqrt{n} r\right)$. Take $N=$ $n+1$ and $m=0$ in Lemma 15; then, we can get

$$
\begin{aligned}
J_{1} & \leq C \sum_{k=1}^{k_{0}} \int_{Q} \frac{2^{n k}}{\left|Q_{k}\right|} \int_{2^{k} \sqrt{n} r \leq\left|y-x_{1}\right|<2^{k+1} \sqrt{n} r} \frac{|f(y)|}{|x-y|^{n+1}} \\
\cdot & |x-y|^{n+1} \\
\cdot & \left|\int_{R^{n}} a(x, \xi) e^{2 \pi i(x-y) \cdot \xi} \phi\left(2^{-j} \xi\right) d \xi\right| d y d x \\
& \leq C \sum_{k=1}^{k_{0}} \int_{Q} \frac{2^{n k}}{\left|Q_{k}\right|} \\
\cdot & \int_{2^{k} \sqrt{n} r \leq\left|y-x_{1}\right|<2^{k+1} \sqrt{n} r} \frac{|f(y)|}{|x-y|^{n+1}} 2^{-j} d y d x \\
& \leq C 2^{-j} \sum_{k=1}^{k_{0}} \int_{Q} \frac{2^{n k}}{\left|Q_{k}\right|} \int_{\left|y-x_{1}\right| 2^{k+1} \sqrt{n} r} \frac{|f(y)|}{\left(2^{k} \sqrt{n} r\right)^{n+1}} d y d x \\
& \leq C \sum_{k=1}^{k_{0}} 2^{-j} 2^{-k} r^{-1} \frac{1}{\left|Q_{k}\right|} \int_{Q_{k}}|f(y)| d y \\
& \leq C 2^{-j} r^{-1} M_{\varphi, \eta} f\left(x_{0}\right) .
\end{aligned}
$$

Take $N=n+n \eta \alpha_{0}+1$ and $m=0$ in Lemma 15; then, we have

$$
\begin{aligned}
J_{2} & \leq C \sum_{k=k_{0}+1}^{\infty} \int_{Q} \frac{2^{n k}}{\left|Q_{k}\right|} \int_{2^{k} \sqrt{n} r \leq\left|y-x_{1}\right|<2^{k+1} \sqrt{n} r} \frac{|f(y)|}{|x-y|^{n+n \eta \alpha_{0}+1}} \\
& \cdot|x-y|^{n+n \eta \alpha_{0}+1} \\
& \cdot\left|\int_{R^{n}} a(x, \xi) e^{2 \pi i(x-y) \cdot \xi} \phi\left(2^{-j} \xi\right) d \xi\right| d y d x \\
& \leq C \sum_{k=k_{0}+1}^{\infty} \int_{Q} \frac{2^{n k}}{\left|Q_{k}\right|} \\
& \cdot \int_{2^{k} \sqrt{n} r \leq\left|y-x_{1}\right|<2^{k+1} \sqrt{n} r} \frac{|f(y)|}{|x-y|^{n+n \eta \alpha_{0}+1}} 2^{-j\left(n \eta \alpha_{0}+1\right)} d y d x \\
& \leq C \sum_{k=k_{0}+1}^{\infty} 2^{-j\left(n \eta \alpha_{0}+1\right)-k\left(n \eta \alpha_{0}+1\right)} r^{-n \eta \alpha_{0}-1} \frac{\varphi\left(\left|Q_{k}\right|\right)^{\eta}}{\varphi\left(\left|Q_{k}\right|\right)^{\eta}\left|Q_{k}\right|} \\
& \cdot \int_{Q_{k}}|f(y)| d y \leq C 2^{-j} r^{-1} M_{\varphi, \eta} f\left(x_{0}\right) .
\end{aligned}
$$

From the above, we know that when $2^{j} r \geq 1$,

$$
\begin{aligned}
& \frac{1}{|Q|} \int_{Q}\left|A_{j} f_{2}(x)-\left(A_{j} f_{2}\right)_{Q}\right| d x \\
& \quad \leq C 2^{-j} r^{-1} M_{\varphi, \eta} f\left(x_{0}\right) .
\end{aligned}
$$

Subcase 2. If $2^{j} r<1$, then also take $k_{0} \in Z$ such that $1 / 2<$ $2^{k_{0}} r \leq 1$. Write

$$
\begin{aligned}
& {\left[a(x, \xi) e^{2 \pi i(x-y) \cdot \xi}-a(z, \xi) e^{2 \pi i(z-y) \cdot \xi}\right]=\sum_{l=1}^{n}\left(x_{l}-z_{l}\right)} \\
& \quad \cdot \int_{0}^{1}\left(\frac{\partial a}{\partial x_{l}}(x(t), \xi) e^{2 \pi i(x(t)-y) \cdot \xi}\right. \\
& \left.\quad+2 \pi i \xi_{l} a(x(t), \xi) e^{2 \pi i(x(t)-y) \cdot \xi}\right) d t
\end{aligned}
$$

where $x(t)=z+t(x-z)$.

The following facts can be checked easily:
(a) $\partial a / \partial x_{l} \in S_{1, \delta}^{\delta}$, where $0<\delta<1$.
(b) $\xi_{l} a(x, \xi) \in S_{1, \delta}^{1}$, where $0<\delta<1$.
(c) $\left|x_{l}-z_{l}\right| \leq r$ for both $x$ and $z$ in $Q$.
(d) If $2^{k} \sqrt{n} r \leq\left|y-x_{1}\right| \leq 2^{k+1} \sqrt{n} r$, since $x(t) \in Q$, then $2^{k-1} \sqrt{n} r \leq|x(t)-y| \leq 2^{k+2} \sqrt{n} r$.

Applying Lemma 15, then we have 


$$
\begin{aligned}
& \frac{1}{|Q|} \int_{Q}\left|A_{j} f_{2}(x)-\left(A_{j} f_{2}\right)_{Q}\right| d x \leq \frac{1}{|Q|} \\
& \cdot \int_{\mathrm{Q}}\left|\frac{1}{|Q|} \int_{\mathrm{Q}} \int_{R^{n}} f_{2}(y) \int_{R^{n}}\left[a(x, \xi) e^{2 \pi i(x-y) \cdot \xi}-a(z, \xi) e^{2 \pi i(z-y) \cdot \xi}\right] \phi\left(2^{-j} \xi\right) d \xi d y d z\right| d x \leq \frac{1}{|Q|} \int_{\mathrm{Q}} \frac{1}{|Q|} \\
& \int_{Q} \int_{R^{n}}\left|f_{2}(y)\right| \sum_{l=1}^{n}\left|x_{l}-z_{l}\right| \\
& \cdot\left|\int_{0}^{1} \int_{R^{n}} \phi\left(2^{-j} \xi\right)\left(\frac{\partial a}{\partial x_{l}}(x(t), \xi) e^{2 \pi i(x(t)-y) \cdot \xi}+2 \pi i \xi_{l} a(x(t), \xi) e^{2 \pi i(x(t)-y) \cdot \xi}\right) d \xi d t\right| d y d z d x \leq C \sum_{k=1}^{k_{0}} \frac{1}{|Q|} \\
& \cdot \int_{Q} \frac{1}{|Q|} \int_{Q} \int_{2^{k} \sqrt{n} r \leq\left|y-x_{1}\right|<2^{k+1} \sqrt{n} r} \frac{|f(y)|}{\left|x_{l}-y\right|^{n+1 / 2}} \sum_{l=1}^{n}\left|x_{l}-z_{l}\right| \int_{0}^{1}|x(t)-y|^{n+1 / 2} \\
& \cdot\left|\int_{R^{n}} \phi\left(2^{-j} \xi\right)\left(\frac{\partial a}{\partial x_{l}}(x(t), \xi) e^{2 \pi i(x(t)-y) \cdot \xi}+2 \pi i \xi_{l} a(x(t), \xi) e^{2 \pi i(x(t)-y) \cdot \xi}\right) d \xi\right| d t d y d z d x+C \sum_{k=k_{0}+1}^{\infty} \frac{1}{|Q|} \\
& \cdot \int_{Q} \frac{1}{|Q|} \int_{Q} \int_{2^{k} \sqrt{n} r \leq\left|y-x_{1}\right|<2^{k+1} \sqrt{n} r} \frac{|f(y)|}{\left|x_{l}-y\right|^{n+1 / 2+n \eta \alpha_{0}}} \sum_{l=1}^{n}\left|x_{l}-z_{l}\right| \int_{0}^{1}|x(t)-y|^{n+1 / 2+n \eta \alpha_{0}} \\
& \cdot\left|\int_{R^{n}} \phi\left(2^{-j} \xi\right)\left(\frac{\partial a}{\partial x_{l}}(x(t), \xi) e^{2 \pi i(x(t)-y) \cdot \xi}+2 \pi i \xi_{l} a(x(t), \xi) e^{2 \pi i(x(t)-y) \cdot \xi}\right) d \xi\right| d t d y d z d x \leq C \sum_{k=1}^{k_{0}} \frac{1}{|Q|} \\
& \cdot \int_{Q} \frac{1}{|Q|} \int_{Q} \int_{2^{k} \sqrt{n} r \leq\left|y-x_{1}\right|<2^{k+1} \sqrt{n} r} \frac{|f(y)|}{\left|x_{l}-y\right|^{n+1 / 2}} \sum_{l=1}^{n}\left|x_{l}-z_{l}\right| \int_{0}^{1} 2^{j / 2} d t d y d z d x+C \sum_{k=k_{0}+1}^{\infty} \frac{1}{|Q|} \int_{Q} \frac{1}{|Q|} \\
& \cdot \int_{Q} \int_{2^{k} \sqrt{n} r \leq\left|y-x_{1}\right|<2^{k+1} \sqrt{n} r} \frac{|f(y)|}{\left|x_{l}-y\right|^{n+1 / 2+n \eta \alpha_{0}}} \sum_{l=1}^{n}\left|x_{l}-z_{l}\right| \int_{0}^{1} 2^{j\left(1 / 2-n \eta \alpha_{0}\right)} d t d y d z d x \leq C \sum_{k=1}^{k_{0}} \frac{1}{|Q|} \int_{Q} \frac{1}{|Q|} \\
& \cdot \int_{Q} \int_{Q_{k}} \frac{|f(y)|}{\left(2^{k} \sqrt{n} r\right)^{n+1 / 2}} n r 2^{j / 2} d y d z d x+C \sum_{k=k_{0}+1}^{\infty} \frac{1}{|Q|} \int_{Q} \frac{1}{|Q|} \int_{Q} \int_{Q_{k}} \frac{|f(y)|}{\left(2^{k} \sqrt{n} r\right)^{n+1 / 2+n \eta \alpha_{0}}} n r 2^{j\left(1 / 2-n \eta \alpha_{0}\right)} d y d z d x \\
& \leq C \sum_{k=1}^{k_{0}} 2^{-k / 2+j / 2} r^{1 / 2} \frac{\left[1+\left(2^{k+2} \sqrt{n} r\right)^{n}\right]^{\alpha_{0} \eta}}{\varphi\left(\left|Q_{k}\right|\right)^{\eta}\left|Q_{k}\right|} \int_{Q_{k}}|f(y)| d y+C \sum_{k=k_{0}+1}^{\infty} 2^{\left(-1 / 2-n \eta \alpha_{0}\right) k} 2^{j\left(1 / 2-n \eta \alpha_{0}\right)} r^{1 / 2-n \eta \alpha_{0}} \frac{\left[1+\left(2^{k+2} \sqrt{n} r\right)^{n}\right]^{\alpha_{0} \eta}}{\varphi\left(\left|Q_{k}\right|\right)^{\eta}\left|Q_{k}\right|} \\
& \cdot \int_{Q_{k}}|f(y)| d y \leq C 2^{j / 2} r^{1 / 2} M_{\varphi, \eta} f\left(x_{0}\right) \sum_{k=1}^{\infty} 2^{-k} \leq C 2^{j / 2} r^{1 / 2} M_{\varphi, \eta} f\left(x_{0}\right) .
\end{aligned}
$$

Putting the two subcases together, we get

$$
\begin{aligned}
& \sum_{j=1}^{\infty} \frac{1}{|Q|} \int_{Q}\left|A_{j} f_{2}(x)-\left(A_{j} f_{2}\right)_{Q}\right| d x \\
& \quad \leq C\left(\sum_{2^{j} r \geq 1} 2^{-j} r^{-1}+\sum_{2^{j} r<1} 2^{j / 2} r^{1 / 2}\right) M_{\varphi, \eta} f\left(x_{0}\right) \\
& \quad \leq C M_{\varphi, \eta} f\left(x_{0}\right) .
\end{aligned}
$$

Case 2. When $r \geq 1$, write $\rho_{1}=\eta / s>\eta$; then,

$$
\begin{aligned}
& \left(\frac{1}{\varphi(|Q|)^{\eta}|Q|} \int_{Q}|T f(x)|^{s} d x\right)^{1 / s} \\
& \leq C \frac{1}{\varphi(|Q|)^{\rho_{1}}}\left(\frac{1}{|Q|} \int_{Q}\left|T f_{1}(x)\right|^{s} d x\right)^{1 / s} \\
& \quad+C \frac{1}{\varphi(|Q|)^{\rho_{1}}}\left(\frac{1}{|Q|} \int_{Q}\left|T f_{2}(x)\right|^{s} d x\right)^{1 / s} \\
& :=J_{3}+J_{4} .
\end{aligned}
$$


Similar to the estimates for $H_{1}$, by Kolmogorov inequality, we get

$$
\begin{aligned}
J_{3} & \leq C \frac{1}{\varphi(|Q|)^{\rho_{1}}|Q|^{1 / s}}\left(|Q|^{1-s}\left\|f_{1}\right\|_{1}^{s}\right)^{1 / s} \\
& =C \frac{1}{\varphi(|Q|)^{\rho_{1}}|Q|} \int_{\bar{Q}}|f(y)| d y \leq C M_{\varphi, \eta} f\left(x_{0}\right) .
\end{aligned}
$$

By Lemmas 14 and 15, we have

$$
\begin{aligned}
& J_{4} \leq C \frac{1}{\varphi(|Q|)^{\rho_{1}}|Q|} \int_{Q}\left|T f_{2}(y)\right| d y \leq C \frac{1}{\varphi(|Q|)^{\rho_{1}}|Q|} \\
& \cdot \int_{Q}\left|A f_{2}(y)\right| d y+C \frac{1}{\varphi(|Q|)^{\rho_{1}}|Q|} \\
& \cdot \sum_{j=1}^{\infty} \int_{Q}\left|A_{j} f_{2}(y)\right| d y \leq C M_{\varphi, \eta}^{\sharp, \Delta}\left(A f_{2}\right)\left(x_{0}\right) \\
& +C \sum_{j=1}^{\infty} \frac{1}{\varphi(|Q|)^{\rho_{1}}|Q|} \int_{Q} \int_{R^{n}}\left|f_{2}(y)\right| \\
& \cdot\left|\int_{R^{n}} a(x, \xi) \phi\left(2^{-j} \xi\right) e^{2 \pi i(x-y) \cdot \xi} d \xi\right| d y d x \\
& \leq C M_{\varphi, \eta} f_{2}\left(x_{0}\right)+C \sum_{j=1}^{\infty} \sum_{k=1}^{\infty} \frac{1}{\varphi(|Q|)^{\rho_{1}}|Q|} \\
& \cdot \int_{Q} \int_{2^{k} \sqrt{n} r \leq\left|y-x_{1}\right|<2^{k+1} \sqrt{n} r} \frac{|f(y)|}{|x-y|^{n+n \eta \alpha_{0}+1}} \\
& \cdot|x-y|^{n+n \eta \alpha_{0}+1} \\
& \cdot\left|\int_{R^{n}} a(x, \xi) \phi\left(2^{-j} \xi\right) e^{2 \pi i(x-y) \cdot \xi} d \xi\right| d y d x \\
& \leq C M_{\varphi, \eta} f\left(x_{0}\right)+C \sum_{j=1}^{\infty} \sum_{k=1}^{\infty} \frac{1}{\varphi(|Q|)^{\rho_{1}}|Q|} \\
& \cdot \int_{Q} \int_{Q_{k}} \frac{|f(y)|}{\left(2^{k} \sqrt{n} r\right)^{n+n \eta \alpha_{0}+1}} 2^{j\left(-n \eta \alpha_{0}-1\right)} d y d x \\
& \leq C M_{\varphi, \eta} f\left(x_{0}\right)+C\left(\sum_{j=1}^{\infty} r^{-1} 2^{-j}\right) M_{\varphi, \eta} f\left(x_{0}\right) \\
& \leq C M_{\varphi, \eta} f\left(x_{0}\right) .
\end{aligned}
$$

From the above we know that when the symbol $a(x, \xi)$ of $T \in L_{1, \delta}^{0}(0<\delta<1)$ has compact $\xi$-support, the inequality

$$
M_{s, \varphi, \eta}^{\sharp, \Delta}(T f)\left(x_{0}\right) \leq C M_{\varphi, \eta} f\left(x_{0}\right)
$$

holds.

When the symbol $a(x, \xi)$ of $T \in L_{1, \delta}^{0}(0<\delta<1)$ has no compact $\xi$-support, let $b_{j}(x, \xi)=a(x, \xi) v\left(2^{-j} \xi\right)$; then, $b_{j}(x, \xi) \rightarrow a(x, \xi)$ as $j \rightarrow \infty$ and $b_{j}(x, \xi) \in S_{1, \delta}^{0}$. Suppose $b_{j}(x, \xi)$ is the symbol of the pseudodifferential operator
$B_{j}$; then, $B_{j} \in L_{1, \delta}^{0}$. Through the dominated convergence theorem, we can achieve $B_{j} f(x) \rightarrow T f(x)$ for all $x$. And by the application of the dominated convergence theorem, we get, for each cube $Q$,

$$
\begin{aligned}
& \left(\left.\frac{1}{|Q|} \int_{Q}|| B_{j} f(x)\right|^{s}-\left|\left(B_{j} f_{2}\right)_{\mathrm{Q}}\right|^{s} \mid d x\right)^{1 / s} \\
& \longrightarrow\left(\left.\frac{1}{|Q|} \int_{Q}|| T f(x)\right|^{s}-\left|\left(T f_{2}\right)_{\mathrm{Q}}\right|^{s} \mid d x\right)^{1 / s}, \\
& \left(\frac{1}{\varphi(|Q|)^{\eta}|Q|} \int_{Q}\left|B_{j} f(x)\right|^{s} d x\right)^{1 / s} \\
& \longrightarrow\left(\frac{1}{\varphi(|Q|)^{\eta}|Q|} \int_{Q}|T f(x)|^{s} d x\right)^{1 / s},
\end{aligned}
$$

as $j \rightarrow \infty$. Applying the previous results to $B_{j}$ and taking the limit as $j \rightarrow \infty$, it is easy to get that

$$
\begin{aligned}
& \left(\left.\frac{1}{|Q|} \int_{Q}|| T f(x)\right|^{s}-\left|\left(T f_{2}\right)_{Q}\right|^{s} \mid d x\right)^{1 / s} \\
& \quad \leq C M_{\varphi, \eta} f\left(x_{0}\right),
\end{aligned}
$$

if $r<1$, and

$$
\left(\frac{1}{\varphi(|Q|)^{\eta}|Q|} \int_{Q}|T f(x)|^{s} d x\right)^{1 / s} \leq C M_{\varphi, \eta} f\left(x_{0}\right),
$$

if $r \geq 1$. Thus, the proof of this lemma is completed.

Lemma 17 (see [12]). If $\omega \in A_{p}(\varphi), 1<p<\infty$, $p^{\prime}=p /(p-$ $1)$, then there exists a constant $C>0$ such that

$$
\left\|M_{\varphi, p^{\prime}} f\right\|_{L^{p}(\omega)} \leq C\|f\|_{L^{p}(\omega)} .
$$

Lemma 18 (see [23]). Let $\omega \in A_{\infty}(\varphi), 1<p<\infty, 0<\eta<$ $\infty$, and $s>0$.

(a) Let $\psi:(0, \infty) \rightarrow(0, \infty)$ be doubling; that is, $\psi(2 a) \leq C \psi(a)$ for $a>0$; then there exists a constant $C>0$ depending upon the $A_{\infty}(\varphi)$ condition of $\omega$ and doubling condition of $\psi$ such that

$$
\begin{aligned}
& \sup _{\lambda>0} \psi(\lambda) \omega\left(\left\{y \in R^{n}: M_{s, \varphi, \eta}^{\Delta} f(y)>\lambda\right\}\right) \\
& \quad \leq C \sup _{\lambda>0} \psi(\lambda) \omega\left(\left\{y \in R^{n}: M_{s, \varphi, \eta}^{\sharp, \Delta} f(y)>\lambda\right\}\right),
\end{aligned}
$$

for every function such that the left-hand side is finite.

(b) Let $f \in L^{p}(\omega), 0<s<p$; then,

$$
\|f\|_{L^{p}(\omega)} \leq\left\|M_{s, \varphi, \eta}^{\Delta} f\right\|_{L^{p}(\omega)} \leq C\left\|M_{s, \varphi, \eta}^{\sharp, \Delta} f\right\|_{L^{p}(\omega)} .
$$

Lemma 19. Let $0<\eta<\infty$; then, there exists a constant $C>0$ such that

$$
M_{\varphi, \eta}^{\sharp, \Delta} f(x) \leq C M_{\varphi, \eta} f(x) .
$$


Proof. For any dyadic cube $Q=Q\left(x_{0}, r\right) \ni x$, we consider two cases about $r$.

Case 1. Consider $0<r<1$. Since $\varphi(|Q|)^{\eta}=(1+|Q|)^{\alpha_{0} \eta} \leq 2^{\alpha_{0} \eta}$, then

$$
\begin{aligned}
& \frac{1}{|Q|} \int_{Q}\left|f(y)-f_{Q}\right| d y \leq \frac{2}{|Q|} \int_{Q}|f(y)| d y \\
& \quad \leq C \frac{1}{\varphi(|Q|)^{\eta}|Q|} \int_{Q}|f(y)| d y \leq C M_{\varphi, \eta} f(x) .
\end{aligned}
$$

Case 2. Consider $r \geq 1$. We have

$$
\frac{1}{\varphi(|Q|)^{\eta}|Q|} \int_{Q\left(x_{0}, r\right)}|f(y)| d y \leq M_{\varphi, \eta} f(x) .
$$

Thus, we can obtain

$$
M_{\varphi, \eta}^{\sharp, \Delta} f(x) \leq C M_{\varphi, \eta} f(x) .
$$

Lemma 20. Let $0<s<1$ and $0<\eta<\infty$; then, there exists a constant $C>0$ such that

$$
M_{s, \varphi, \eta}^{\sharp, \Delta} f(x) \leq C M_{\varphi, \eta} f(x) .
$$

Proof. For any dyadic cube $Q=Q\left(x_{0}, r\right) \ni x$, it follows from $0<s<1$ that $1 / \varphi(|Q|)^{\eta / s}<1 / \varphi(|Q|)^{\eta}$. From Lemma 19, we have

$$
\begin{aligned}
M_{s, \varphi, \eta}^{\sharp \Delta} f(x) & =M_{\varphi, \eta}^{\sharp, \Delta}\left(|f|^{s}\right)^{1 / s}(x) \\
& \leq C\left[M_{\varphi, \eta}\left(|f|^{s}\right)(x)\right]^{1 / s} \\
& \leq C \sup _{\mathrm{Q} \ni x \varphi} \frac{1}{\varphi(|Q|)^{\eta / s}|Q|} \int_{Q}|f(y)| d y \\
& \leq C \sup _{Q \ni x} \frac{1}{\varphi(|Q|)^{\eta}|Q|} \int_{Q}|f(y)| d y \\
& =C M_{\varphi, \eta} f(x) .
\end{aligned}
$$

Lemma 21 (see [12]). Let $\omega \in A_{\infty}(\varphi)$ and $0<\eta<\infty$. For any $f \in L_{l o c}^{1}\left(R^{n}\right)$ and $0<\gamma<b<b_{0}=1 / \varphi\left(2^{n}\right)^{\eta}$, there exist constants $C>0$ and $\delta_{1}$ such that

$$
\begin{gathered}
\omega\left(\left\{x \in R^{n}: M_{\varphi, \eta}^{\Delta} f(x)>\lambda, M_{\varphi, \eta}^{\sharp, \Delta} f(x) \leq \gamma \lambda\right\}\right) \\
\leq C a^{\delta_{1}} \omega\left(\left\{x \in R^{n}: M_{\varphi, \eta}^{\Delta} f(x)>b \lambda\right\}\right)
\end{gathered}
$$

for all $\lambda>0$, where $a=2^{n} \gamma /\left(1-b / b_{0}\right)$.

If the pseudodifferential operator $T$ is given with symbol $a(x, \xi) \in S_{\rho, \delta}^{m}$, we can also write it as $T_{a}$; that is,

$$
\begin{aligned}
T f(x) & :=T_{a} f(x)=\int_{R^{n}} a(x, \xi) e^{2 \pi i x \cdot \xi} \widehat{f}(\xi) d \xi \\
& =\int_{R^{n}} K(x, y) f(y) d y,
\end{aligned}
$$

where $K(x, y)=\int_{R^{n}} a(x, \xi) e^{2 \pi i(x-y) \cdot \xi} d \xi$.
Write $T_{a}:=T_{a_{0}}+\sum_{j=1}^{\infty} T_{a_{j}}$, where $a_{0}(x, \xi)=a(x, \xi) \nu(\xi)$, $a_{j}(x, \xi)=a(x, \xi) \phi\left(2^{-j} \xi\right), j=1,2, \ldots$; then,

$$
\begin{aligned}
T_{a_{j}} f(x) & =\int_{R^{n}} a_{j}(x, \xi) e^{2 \pi i x \cdot \xi} \hat{f}(\xi) d \xi \\
& =\int_{R^{n}} K_{j}(x, x-y) f(y) d y \\
& =\int_{R^{n}} K_{j}(x, z) f(x-z) d z,
\end{aligned}
$$

where $K_{j}(x, z)=\int_{R^{n}} a_{j}(x, \xi) e^{2 \pi i z \cdot \xi} d \xi$ and $K(x, y)=$ $\sum_{j=0}^{\infty} K_{j}(x, x-y)$.

Lemma 22. Let $a \in S_{1, \delta}^{0}, 0<\delta<1$; then, for all multi-indices $\alpha$ and $\beta$, the following estimates

$$
\left|\partial_{x}^{\alpha} \partial_{z}^{\beta} K_{j}(x, z)\right| \leq C_{M, \alpha, \beta}|z|^{-M} 2^{j(n-M+|\beta|+\delta|\alpha|)}
$$

hold for every integer $M \geq 0$, where $C_{M, \alpha, \beta}$ is independent of $j \geq 0$.

Proof. Write

$$
\begin{gathered}
\int_{R^{n}} \partial_{\xi}^{\gamma}\left[(2 \pi i \xi)^{\beta} \partial_{x}^{\alpha} a_{j}(x, \xi)\right] e^{2 \pi i \xi \cdot z} d \xi \\
=(-2 \pi i z)^{\gamma} \partial_{x}^{\alpha} \partial_{z}^{\beta}\left[K_{j}(x, z)\right] .
\end{gathered}
$$

When $j=0$, for $\nu(\xi)$ supported in $\{\xi:|\xi| \leq 2\}$ and $\nu(\xi) \epsilon$ $C_{0}^{\infty}\left(R^{n}\right)$, by taking $|\gamma|=M$, we have

$$
\begin{aligned}
& \left|(-2 \pi i z)^{\gamma} \partial_{x}^{\alpha} \partial_{z}^{\beta}\left(K_{0}(x, z)\right)\right| \\
& \quad=\left|\int_{R^{n}} \partial_{\xi}^{\gamma}\left[(2 \pi i \xi)^{\beta} \partial_{x}^{\alpha} a_{0}(x, \xi)\right] e^{2 \pi i \xi \cdot z} d \xi\right| \\
& \quad=\left|\int_{|\xi| \leq 2} \partial_{\xi}^{\gamma}\left[(2 \pi i \xi)^{\beta}\left(\partial_{x}^{\alpha} a(x, \xi)\right) \nu(\xi)\right] e^{2 \pi i \xi \cdot z} d \xi\right| \\
& \quad \leq C_{M, \alpha, \beta} .
\end{aligned}
$$

When $j \geq 1$, for $\phi\left(2^{j} \xi\right)$ supported in $\left\{\xi: 2^{j-1} \leq|\xi| \leq\right.$ $\left.2^{j+1}\right\}$ and $1+|\xi| \sim 2^{j}$, by taking $|\gamma|=M$, we have

$$
\begin{aligned}
\left|(-2 \pi i z)^{\gamma} \partial_{x}^{\alpha} \partial_{z}^{\beta}\left[K_{j}(x, z)\right]\right| \\
=\left|\int_{R^{n}} \partial_{\xi}^{\gamma}\left[(2 \pi i \xi)^{\beta} \partial_{x}^{\alpha} a_{j}(x, \xi)\right] e^{2 \pi i \xi \cdot z} d \xi\right| \\
=\mid \int_{2^{j-1} \leq|\xi| \leq 2^{j+1}} \partial_{\xi}^{\gamma}\left[(2 \pi i \xi)^{\beta}\left(\partial_{x}^{\alpha} a(x, \xi)\right) \phi\left(2^{-j} \xi\right)\right] \\
\cdot e^{2 \pi i \xi \cdot z} d \xi \mid \leq C_{M, \alpha, \beta} 2^{j(n-M+|\beta|+\delta|\alpha|)} .
\end{aligned}
$$

From all the above, we can obtain that, for any $j \geq 0$,

$$
\left|\partial_{x}^{\alpha} \partial_{z}^{\beta} K_{j}(x, z)\right| \leq C_{M, \alpha, \beta}|z|^{-M} 2^{j(n-M+|\beta|+\delta|\alpha|)} .
$$


Lemma 23. Let $a \in S_{1, \delta}^{0}, 0<\delta<1$, and $K(x, y) \in C^{\infty}\left(R^{n} \times\right.$ $\left.\left(R^{n} \backslash\{0\}\right)\right)$ be the kernel of $a(x, \xi)$; then, for all $N \geq 0$, the following inequalities hold:

$$
\begin{gathered}
|K(x, y)| \leq C_{N}|x-y|^{-n-N}, \quad x \neq y ; \\
\left|\partial_{x} K(x, y)\right| \leq C_{N}|x-y|^{-n-N-1}, \quad x \neq y .
\end{gathered}
$$

Proof. Write

$$
\begin{aligned}
K(x, y) & =\sum_{j=0}^{\infty} K_{j}(x, x-y), \\
\partial_{x} K(x, y) & =\sum_{j=0}^{\infty} \partial_{x}\left[K_{j}(x, x-y)\right] .
\end{aligned}
$$

Put $z=x-y$; then,

$$
\begin{aligned}
\partial_{x} K(x, y) \\
\quad=\left.\sum_{j=0}^{\infty}\left[\left(\partial_{x} K_{j}\right)(x, z)+\left(\partial_{z} K_{j}\right)(x, z)\right]\right|_{z=x-y} .
\end{aligned}
$$

It follows from Lemma 22 that, for every integer $M \geq 0$,

$$
\begin{aligned}
\left|\partial_{x} K(x, y)\right| \leq & \sum_{j=0}^{\infty} C_{M}|x-y|^{-M} 2^{j(n-M+\delta)} \\
& +\sum_{j=0}^{\infty} C_{M}|x-y|^{-M} 2^{j(n-M+1)} \\
\leq & \sum_{j=0}^{\infty} C_{M}|x-y|^{-M} 2^{j(n-M+1)} .
\end{aligned}
$$

Case 1. When $0<|x-y| \leq 1$,

$$
\begin{aligned}
\left|\partial_{x} K(x, y)\right| \leq & \sum_{2^{j} \leq|x-y|^{-1}} C_{M}|x-y|^{-M} 2^{j(n-M+1)} \\
& +\sum_{2^{j}>|x-y|^{-1}} C_{M}|x-y|^{-M} 2^{j(n-M+1)} \\
:= & K_{1}+K_{2} .
\end{aligned}
$$

First, we estimate $K_{1}$. Take $M=0$ and $k$ is the biggest integer no more than $\log _{2}|x-y|^{-1}$; then,

$$
K_{1}=\sum_{j=0}^{k} C 2^{j(n+1)} \leq C|x-y|^{-n-N-1} .
$$

Next, we estimate $K_{2}$. By taking $M>n+1$, we have

$$
\begin{aligned}
K_{2} & =\sum_{j=k+1}^{\infty} C_{M}|x-y|^{-M} 2^{j(n-M+1)} \\
& \leq C_{M}|x-y|^{-M} 2^{\left(\log _{2}|x-y|^{-1}\right)(n-M+1)} \\
& \leq C|x-y|^{-n-N-1} .
\end{aligned}
$$

From the above we can obtain that when $0<|x-y| \leq 1$,

$$
\left|\partial_{x} K(x, y)\right| \leq C_{N}|x-y|^{-n-N-1}, \quad x \neq y .
$$

Case 2. When $|x-y|>1$, by taking $M>n+1+N$, we get

$$
\begin{aligned}
\left|\partial_{x} K(x, y)\right| & \leq \sum_{j=0}^{\infty} C_{M}|x-y|^{-M} 2^{j(n-M+1)} \\
& =C_{M}|x-y|^{-M} \frac{1}{1-2^{n-M+1}} \\
& \leq C_{N}|x-y|^{-n-N-1} .
\end{aligned}
$$

Thus, for all $N \geq 0$,

$$
\left|\partial_{x} K(x, y)\right| \leq C_{N}|x-y|^{-n-N-1}, \quad x \neq y .
$$

Similarly we can obtain that, for all $N \geq 0$,

$$
|K(x, y)| \leq C_{N}|x-y|^{-n-N}, \quad x \neq y .
$$

Thus, we complete the proof of the lemma.

Lemma 24 (see [23]). If $g \in L_{l o c}^{1}\left(R^{n}\right)$ and supp $g \subset \mathrm{Q}$, then

$$
\frac{1}{|Q|} \int_{Q} M g(y) d y \leq C\|g\|_{L \log L, Q} \text {. }
$$

Lemma 25. Let $0<\eta<\infty$; then, there exists a constant $C>0$ such that

$$
M_{\varphi, \eta} f(x) \leq C M_{L \log L, \varphi, \eta} f(x) .
$$

Proof. For all $Q \ni x$, by Lebesgue differential theorem and Lemma 24, we have

$$
\begin{aligned}
& \frac{1}{\varphi(|Q|)^{\eta}|Q|} \int_{Q}|f(y)| d y \\
& =\frac{1}{\varphi(|Q|)^{\eta}|Q|} \int_{Q}\left|f \chi_{Q}(y)\right| d y \\
& \leq \frac{1}{\varphi(|Q|)^{\eta}|Q|} \int_{Q} M\left(f \chi_{Q}\right)(y) d y \\
& \leq C \varphi(|Q|)^{-\eta}\left\|f \chi_{Q}\right\|_{L \log L, Q} \\
& \quad \leq C \varphi(|Q|)^{-\eta}\|f\|_{L \log L, Q} .
\end{aligned}
$$

Then, taking supremum for all $Q \ni x$, we can have the estimates

$$
M_{\varphi, \eta} f(x) \leq C M_{L \log L, \varphi, \eta} f(x) .
$$

Lemma 26 (see [12]). Let $0<\eta<\infty$ and $M_{\varphi, \eta / 2}(f) \in$ $L_{\text {loc }}^{1}\left(R^{n}\right)$; then, there exist constants $C_{1}>0$ and $C_{2}>0$, which are independent of $f$ and $x$, such that

$$
\begin{aligned}
C_{1} M_{\varphi, \eta} M_{\varphi, \eta} f(x) & \leq M_{L \log L, \varphi, \eta} f(x) \\
& \leq C_{2} M_{\varphi, \eta / 2} M_{\varphi, \eta / 2} f(x) .
\end{aligned}
$$


Lemma 27. Let $m \in \mathbb{N}, T_{j, 1} \in L_{1, \delta}^{0}, 0<\delta<1$, or $T_{j, 1}= \pm I$, $j=1,2, \ldots, m, b \in B M O, 0<\eta<\infty$, and $0<s<\varepsilon<1$. Suppose $T_{1}(f)=0$ when $f \in C_{0}^{\infty}\left(R^{n}\right)$. Then, for all $x \in R^{n}$,

$$
\begin{aligned}
& M_{s, \varphi, \eta}^{\sharp, \Delta}\left(T_{b} f\right)(x) \leq C\|b\|_{B M O} \\
& \cdot \sum_{j=1}^{m}\left(M_{L \log L, \varphi, \eta}\left(T_{j, 2} f\right)(x)+M_{\varepsilon, \varphi, \eta}^{\Delta}\left(T_{j, 2} f\right)(x)\right) .
\end{aligned}
$$

Proof. For fixed $x \in R^{n}$, let any dyadic cube $Q=Q\left(x_{0}, r\right) \ni x$ and $\bar{Q}=Q\left(x_{0}, 4 \sqrt{n} r\right)$. Since $f \in C_{0}^{\infty}\left(R^{n}\right), T_{1}(f)=0$, then

$$
\begin{aligned}
T_{b} f(x) & =T_{\left(b-b_{\bar{Q}}\right)} f(x) \\
& =T_{\left(\left(b-b_{\overline{\mathrm{Q}}}\right) \chi_{\overline{\mathrm{Q}}}\right)} f(x)+T_{\left(\left(b-b_{\overline{\mathrm{Q}}}\right) \chi_{(\overline{\mathrm{Q}})^{c}}\right)} f(x) .
\end{aligned}
$$

Write

$$
\begin{aligned}
& M_{s, \varphi, \eta}^{\sharp, \Delta}\left(T_{b} f\right)(x) \\
& \leq C \sup _{\mathrm{Q} \ni x, r<1}\left(\left.\inf _{c} \frac{1}{|Q|} \int_{Q}|| T_{b} f(y)\right|^{s}-c \mid d y\right)^{1 / s} \\
& \quad+C \sup _{\mathrm{Q} \ni x, r \geq 1}\left(\frac{1}{\varphi(|Q|)^{\eta}|Q|} \int_{Q}\left|T_{b} f(y)\right|^{s} d y\right)^{1 / s} .
\end{aligned}
$$

Case 1. When $0<r<1$, take $c_{0}=T_{\left(\left(b-b_{\bar{Q}}\right) \chi_{(\bar{Q})^{c}}\right)} f\left(x_{0}\right)$ :

$$
\begin{aligned}
\inf _{c}\left(\left.\frac{1}{|Q|} \int_{Q}|| T_{b} f(y)\right|^{s}-c \mid d y\right)^{1 / s} \\
\leq\left(\frac{1}{|Q|} \int_{Q}\left|T_{b} f(y)-c_{0}\right|^{s} d y\right)^{1 / s} \\
\leq C\left(\frac{1}{|Q|} \int_{Q}\left|T_{\left(\left(b-b_{\bar{Q}}\right) x_{\bar{Q}}\right)} f(y)\right|^{s} d y\right)^{1 / s} \\
\quad+C\left(\frac{1}{|Q|} \int_{Q}\left|T_{\left(\left(b-b_{\bar{Q}}\right) x_{(\bar{Q})}\right)} f(y)-c_{0}\right|^{s} d y\right)^{1 / s} \\
:=I+I I .
\end{aligned}
$$

Firstly, we estimate $I$ :

$$
\begin{aligned}
I & \left.\leq C \sum_{j=1}^{m}\left(\frac{1}{|Q|} \int_{Q} \mid T_{j, 1} M_{\left(\left(b-b_{\bar{Q}}\right)\right.}\right) T_{\bar{Q}} T_{j, 2} f(y) \mid d y\right)^{1 / s} \\
& :=C \sum_{j=1}^{m} I_{j} .
\end{aligned}
$$

Subcase 1. When $T_{j, 1} \in L_{1, \delta}^{0}, 0<\delta<1$, according to [4, 24], we know that $T_{j, 1}$ is of the weak type $(1,1)$. By Kolmogorov inequality, we get

$$
\begin{aligned}
I_{j} & \leq C|Q|^{-1 / s}\left(|Q|^{1-s}\left\|\left(b-b_{\overline{\mathrm{Q}}}\right) \chi_{\overline{\mathrm{Q}}} T_{j, 2} f\right\|_{1}^{s}\right)^{1 / s} \\
& \leq C \frac{1}{|\overline{\mathrm{Q}}|} \int_{\overline{\mathrm{Q}}}\left|b(y)-b_{\overline{\mathrm{Q}}}\right|\left|T_{j, 2} f(y)\right| d y \\
& \leq C\|b\|_{\mathrm{BMO}}\left\|T_{j, 2} f(y)\right\|_{L \log L, \overline{\mathrm{Q}}} \\
& \leq C\|b\|_{\mathrm{BMO}} M_{L \log L, \varphi, \eta}\left(T_{j, 2} f\right)(x) .
\end{aligned}
$$

Subcase 2. When $T_{j, 1}= \pm I$, take $1<q<\min \{\varepsilon / s, 1 /(1-s)\}$; let $1 / q+1 / q^{\prime}=1$; then, $1<s q^{\prime}<\infty$ and

$$
\begin{aligned}
& \leq\left(\frac{1}{|Q|} \int_{Q}\left|b(y)-b_{\bar{Q}}\right|^{s q^{\prime}} d y\right)^{1 /\left(s q^{\prime}\right)} \\
& \cdot\left(\frac{1}{|Q|} \int_{Q}\left|T_{j, 2} f(y)\right|^{s q} d y\right)^{1 /(s q)} .
\end{aligned}
$$$$
I_{j}=\left(\frac{1}{|Q|} \int_{Q}\left|\left(\left(b(y)-b_{\bar{Q}}\right) \chi_{\bar{Q}}\right) T_{j, 2} f(y)\right|^{s} d y\right)^{1 / s}
$$

Since $\varepsilon /(s q)>1$ and $0<r<1$, then $(1+|Q|)^{\eta \alpha_{0} / \varepsilon} \leq 2^{\eta \alpha_{0} / \varepsilon}$. We have

$$
\begin{aligned}
& \left(\frac{1}{|Q|} \int_{Q}\left|T_{j, 2} f(y)\right|^{s q} d y\right)^{1 /(s q)} \\
& \leq\left(\frac{1}{|Q|} \int_{Q}\left|T_{j, 2} f(y)\right|^{\varepsilon} d y\right)^{1 / \varepsilon} \\
& \leq C M_{\varepsilon, \varphi, \eta}^{\Delta}\left(T_{j, 2} f\right)(x) .
\end{aligned}
$$

By the John-Nirenberg inequality of BMO, we can get that

$$
I_{j} \leq C\|b\|_{\mathrm{BMO}} M_{\varepsilon, \varphi, \eta}^{\Delta}\left(T_{j, 2} f\right)(x) .
$$

Thus,

$$
\begin{aligned}
I \leq & C\|b\|_{\mathrm{BMO}} \\
& \cdot \sum_{j=1}^{m}\left(M_{L \log L, \varphi, \eta}\left(T_{j, 2} f\right)(x)+M_{\varepsilon, \varphi, \eta}^{\Delta}\left(T_{j, 2} f\right)(x)\right) .
\end{aligned}
$$

Secondly, we estimate II:

$$
\begin{aligned}
I I & \leq C \frac{1}{|Q|} \int_{Q} \mid T_{\left(\left(b-b_{\bar{Q}}\right) \chi_{(\bar{Q})^{c}}\right)} f(y) \\
& -T_{\left(\left(b-b_{\bar{Q}}\right) \chi_{\left.(\bar{Q})^{c}\right)}\right.} f\left(x_{0}\right) \mid d y \leq C \sum_{j=1}^{m} \frac{1}{|Q|} \\
& \cdot \int_{Q} \mid T_{j, 1} M_{\left(\left(b-b_{\bar{Q}}\right) \chi_{(\bar{Q})}\right)} T_{j, 2} f(y) \\
& -T_{j, 1} M_{\left(\left(b-b_{\bar{Q}}\right) \chi_{\left.(\bar{Q})^{c}\right)}\right.} T_{j, 2} f\left(x_{0}\right) \mid d y .
\end{aligned}
$$


Let $I I_{j}:=\left|T_{j, 1} M_{\left(\left(b-b_{\bar{Q}}\right) \chi_{(\bar{Q})}\right)^{c}} T_{j, 2} f(y)-T_{j, 1} M_{\left(\left(b-b_{\bar{Q}}\right) \chi_{(\bar{Q})}\right)} T_{j, 2} f\left(x_{0}\right)\right|$.

Subcase 1. When $T_{j, 1} \in L_{1, \delta}^{0}, 0<\delta<1$, we can express that

$$
T_{j, 1} f(x)=\int_{R^{n}} K(x, y) f(y) d y,
$$

where $K(x, y)=\int_{R^{n}} a(x, \xi) e^{2 \pi i(x-y) \cdot \xi} d \xi$ and $a(x, \xi)$ is the symbol of $T_{j, 1}$. According to Lemma 23 , we know that $K(x, y)$ satisfies the following inequality:

$$
\left|\partial_{x} K(x, y)\right| \leq C_{N}|x-y|^{-n-N-1}, \quad x \neq y,
$$

for all $N \geq 0$.

Take $k_{0}$ such that $1 / 2<2^{k_{0}} r \leq 1$. We get

$$
\begin{aligned}
I I_{j} & \leq \int_{(\overline{\mathrm{Q}})^{c}}\left|K(y, z)-K\left(x_{0}, z\right)\right|\left|b(z)-b_{\overline{\mathrm{Q}}}\right| \\
& \cdot\left|T_{j, 2} f(z)\right| d z \\
& \leq \sum_{k=1}^{k_{0}} \int_{2^{k} \sqrt{n} r \leq\left|x_{0}-z\right|<2^{k+1} \sqrt{n r}}\left|K(y, z)-K\left(x_{0}, z\right)\right| \\
& \cdot\left|b(z)-b_{\overline{\mathrm{Q}}}\right|\left|T_{j, 2} f(z)\right| d z \\
& +\sum_{k=k_{0}+1}^{\infty} \int_{2^{k} \sqrt{n} r \leq\left|x_{0}-z\right|<2^{k+1} \sqrt{n} r}\left|K(y, z)-K\left(x_{0}, z\right)\right| \\
& \cdot\left|b(z)-b_{\overline{\mathrm{Q}}}\right|\left|T_{j, 2} f(z)\right| d z:=I I_{j}^{\prime}+I I_{j}^{\prime \prime} .
\end{aligned}
$$

Now we estimate $I I_{j}^{\prime}$. Let $N=0$ and $Q_{k}=Q\left(x_{0}, 2^{k+2} \sqrt{n} r\right)$. If $h$ is on the line between $y$ and $x_{0}$, then $h \in Q$ and $|h-z| \geq$ $2^{k-1} \sqrt{n} r$. Thus,

$$
\begin{aligned}
\left|K(y, z)-K\left(x_{0}, z\right)\right| & =\left|\partial_{x} K(h, z)\left(y-x_{0}\right)\right| \\
& \leq C|h-z|^{-n-1}\left|y-x_{0}\right| \\
& \leq C 2^{-k}\left|Q_{k}\right|^{-1} .
\end{aligned}
$$

Then, we can get

$$
\begin{aligned}
& I I_{j}^{\prime} \leq C \sum_{k=1}^{k_{0}} \int_{2^{k} \sqrt{n} r \leq\left|x_{0}-z\right|<2^{k+1} \sqrt{n} r} 2^{-k}\left|Q_{k}\right|^{-1}\left|b(z)-b_{\bar{Q}}\right| \\
& \cdot\left|T_{j, 2} f(z)\right| d z \leq C \sum_{k=1}^{k_{0}} \frac{2^{-k}}{\left|Q_{k}\right|} \int_{Q_{k}}\left|b(z)-b_{Q_{k}}\right| \\
& \cdot\left|T_{j, 2} f(z)\right| d z+C \sum_{k=1}^{k_{0}} \frac{2^{-k}}{\left|Q_{k}\right|} \int_{Q_{k}}\left|b_{Q_{k}}-b_{\bar{Q}}\right| \\
& \cdot\left|T_{j, 2} f(z)\right| d z:=I I I_{j}^{\prime}+I I I_{j}^{\prime \prime} .
\end{aligned}
$$

Applying the generalized Hölder inequality,

$$
\frac{1}{|Q|} \int_{Q}|f g| d y \leq\|f\|_{B, Q}\|g\|_{\bar{B}, Q}
$$

and noticing that $\varphi\left(\left|Q_{k}\right|\right)^{\eta} \leq\left(1+(4 \sqrt{n})^{n}\right)^{\alpha_{0} \eta}$ for $k \leq k_{0}$, we have

$$
\begin{aligned}
I I I_{j}^{\prime} & \leq C \sum_{k=1}^{k_{0}} 2^{-k}\|b\|_{\mathrm{BMO}}\left\|T_{j, 2} f\right\|_{L \log L, \mathrm{Q}_{k}} \\
& \leq C\left(\sum_{k=1}^{\infty} 2^{-k}\right)\|b\|_{\mathrm{BMO}} M_{L \log L, \varphi, \eta}\left(T_{j, 2} f\right)(x) \\
& \leq C\|b\|_{\mathrm{BMO}} M_{L \log L, \varphi, \eta}\left(T_{j, 2} f\right)(x) .
\end{aligned}
$$

Since $\left|b_{\mathrm{Q}_{k}}-b_{\overline{\mathrm{Q}}}\right| \leq 2^{n} k\|b\|_{\mathrm{BMO}}$, then by Lemma 25 we have

$$
\begin{aligned}
& I I I_{j}^{\prime \prime} \leq C \sum_{k=1}^{k_{0}} 2^{n} k\|b\|_{\mathrm{BMO}} 2^{-k} \varphi\left(\left|Q_{k}\right|\right)^{\eta} \varphi\left(\left|Q_{k}\right|\right)^{-\eta}\left|Q_{k}\right|^{-1} \\
& \cdot \int_{Q_{k}}\left|T_{j, 2} f(z)\right| d z \leq C\left(\sum_{k=1}^{\infty} 2^{-k} k\right)\|b\|_{\mathrm{BMO}} \\
& \cdot M_{\varphi, \eta}\left(T_{j, 2} f\right)(x) \leq C\|b\|_{\mathrm{BMO}} M_{L \log L, \varphi, \eta}\left(T_{j, 2} f\right) \\
& \cdot(x) .
\end{aligned}
$$

Thus,

$$
I I_{j}^{\prime} \leq C\|b\|_{\mathrm{BMO}} M_{L \log L, \varphi, \eta}\left(T_{j, 2} f\right)(x) .
$$

Next we estimate $I I_{j}^{\prime \prime}$. Take $N=n \eta \alpha_{0}$; similar to the estimate for $I I_{j}^{\prime}$, we get

$$
\begin{aligned}
\left|K(y, z)-K\left(x_{0}, z\right)\right| & =\left|\partial_{x} K(h, z)\left(y-x_{0}\right)\right| \\
& \leq C|h-z|^{-n-n \eta \alpha_{0}-1}\left|y-x_{0}\right| \\
& \leq C 2^{-k}\left|Q_{k}\right|^{-1} \varphi\left(\left|Q_{k}\right|\right)^{-\eta} .
\end{aligned}
$$

Then, we achieve that

$$
\begin{aligned}
& I I_{j}^{\prime \prime} \leq C \sum_{k=k_{0}+1}^{\infty} \int_{2^{k} \sqrt{n} r \leq\left|x_{0}-z\right|<2^{k+1} \sqrt{n} r} 2^{-k}\left|Q_{k}\right|^{-1} \\
& \quad \varphi\left(\left|Q_{k}\right|\right)^{-\eta}\left|b(z)-b_{\bar{Q}}\right|\left|T_{j, 2} f(z)\right| d z \\
& \quad \leq C \sum_{k=k_{0}+1}^{\infty} \frac{2^{-k}}{\varphi\left(\left|Q_{k}\right|\right)^{\eta}\left|Q_{k}\right|} \int_{Q_{k}}\left|b(z)-b_{Q_{k}}\right| \\
& \cdot\left|T_{j, 2} f(z)\right| d z+C \sum_{k=k_{0}+1}^{\infty} \frac{2^{-k}}{\varphi\left(\left|Q_{k}\right|\right)^{\eta}\left|Q_{k}\right|} \\
& \cdot \int_{Q_{k}}\left|b_{Q_{k}}-b_{\bar{Q}}\right|\left|T_{j, 2} f(z)\right| d z:=I V_{j}^{\prime}+I V_{j}^{\prime \prime} .
\end{aligned}
$$


By the generalized Hölder inequality, we get

$$
\begin{aligned}
I V_{j}^{\prime} & \leq C \sum_{k=k_{0}+1}^{\infty} 2^{-k} \varphi\left(\left|Q_{k}\right|\right)^{-\eta}\|b\|_{\mathrm{BMO}}\left\|T_{j, 2} f\right\|_{L \log L, \mathrm{Q}_{k}} \\
& \leq C\left(\sum_{k=1}^{\infty} 2^{-k}\right)\|b\|_{\mathrm{BMO}} M_{L \log L, \varphi, \eta}\left(T_{j, 2} f\right)(x) \\
& \leq C\|b\|_{\mathrm{BMO}} M_{L \log L, \varphi, \eta}\left(T_{j, 2} f\right)(x) .
\end{aligned}
$$

Similar to the estimates for $I I I_{j}^{\prime \prime}$, we can get

$$
\begin{aligned}
& I V_{j}^{\prime \prime} \leq C \sum_{k=k_{0}+1}^{\infty} 2^{-k} 2^{n} k\|b\|_{\mathrm{BMO}} \varphi\left(\left|Q_{k}\right|\right)^{-\eta}\left|Q_{k}\right|^{-1} \\
& \cdot \int_{\mathrm{Q}_{k}}\left|T_{j, 2} f(z)\right| d z \leq C\left(\sum_{k=1}^{\infty} 2^{-k} k\right)\|b\|_{\mathrm{BMO}} \\
& \cdot M_{\varphi, \eta}\left(T_{j, 2} f\right)(x) \leq C\|b\|_{\mathrm{BMO}} M_{L \log L, \varphi, \eta}\left(T_{j, 2} f\right) \\
& \cdot(x) .
\end{aligned}
$$

From the above, we can obtain

$$
I I_{j}^{\prime \prime} \leq C\|b\|_{\mathrm{BMO}} M_{L \log L, \varphi, \eta}\left(T_{j, 2} f\right)(x) .
$$

So

$$
I I_{j} \leq C\|b\|_{\mathrm{BMO}} M_{L \log L, \varphi, \eta}\left(T_{j, 2} f\right)(x) .
$$

Subcase 2. When $T_{j, 1}= \pm I$, we have

$$
\begin{aligned}
I I_{j} & =\mid\left(b(y)-b_{\overline{\mathrm{Q}}}\right) \chi_{(\overline{\mathrm{Q}})^{c}}(y) T_{j, 2} f(y) \\
& -\left(b\left(x_{0}\right)-b_{\overline{\mathrm{Q}}}\right) \chi_{(\overline{\mathrm{Q}})^{c}}\left(x_{0}\right) T_{j, 2} f\left(x_{0}\right) \mid=0 .
\end{aligned}
$$

Thus,

$$
I I \leq C\|b\|_{\text {BMO }} \sum_{j=1}^{m} M_{L \log L, \varphi, \eta}\left(T_{j, 2} f\right)(x) .
$$

So when $r<1$,

$$
\begin{array}{r}
\sup _{\mathrm{Q} \ni x, r<1}\left(\inf _{c} \frac{1}{Q} \int_{Q} \|\left. T_{b} f(y)\right|^{s}-c \mid d y\right)^{1 / s} \leq C\|b\|_{\mathrm{BMO}} \\
\cdot \sum_{j=1}^{m}\left(M_{L \log L, \varphi, \eta}\left(T_{j, 2} f\right)(x)+M_{\varepsilon, \varphi, \eta}^{\Delta}\left(T_{j, 2} f\right)(x)\right) .
\end{array}
$$

Case 2. When $r \geq 1$, write $\rho_{2}=\eta / s>\eta$; then,

$$
\begin{aligned}
& \left(\frac{1}{\varphi(|Q|)^{\eta}|Q|} \int_{Q}\left|T_{b} f(y)\right|^{s} d y\right)^{1 / s} \leq C \sum_{j=1}^{m} \frac{1}{\varphi(|Q|)^{\rho_{2}}} \\
& \cdot\left(\frac{1}{|Q|} \int_{Q}\left|T_{j, 1} M_{\left(b-b_{\bar{Q}}\right)} T_{j, 2} f(y)\right|^{s} d y\right)^{1 / s} \\
& \leq C \sum_{j=1}^{m} \frac{1}{\varphi(|Q|)^{\rho_{2}}} \\
& \cdot\left(\frac{1}{|Q|} \int_{Q}\left|T_{j, 1} M_{\left(b-b_{\bar{Q}}\right) \chi_{\bar{Q}}} T_{j, 2} f(y)\right|^{s} d y\right)^{1 / s} \\
& +C \sum_{j=1}^{m} \frac{1}{\varphi(|Q|)^{\rho_{2}}} \\
& \cdot\left(\frac{1}{|Q|} \int_{Q}\left|T_{j, 1} M_{\left.\left(b-b_{\bar{Q}}\right) \chi_{(\bar{Q})}\right)^{2}} T_{j, 2} f(y)\right|^{s} d y\right)^{1 / s} \\
& :=C \sum_{j=1}^{m} K_{j}^{\prime}+C \sum_{j=1}^{m} K_{j}^{\prime \prime} .
\end{aligned}
$$

Subcase 1. When $T_{j, 1} \in L_{1, \delta}^{0}, 0<\delta<1$, since $T_{j, 1}$ is of the weak type $(1,1)$, by Kolmogorov inequality and the generalized Hölder inequality, we can get

$$
K_{j}^{\prime}
$$

$$
\begin{aligned}
& \leq C \frac{1}{\varphi(|Q|)^{\rho_{2}}}|Q|^{-1 / s}\left(|Q|^{1-s}\left\|\left(b-b_{\bar{Q}}\right) \chi_{\bar{Q}} T_{j, 2} f\right\|_{1}^{s}\right)^{1 / s} \\
& \leq C \frac{1}{\varphi(|Q|)^{\rho_{2}}} \frac{|\bar{Q}|}{|Q|}\|b\|_{\mathrm{BMO}}\left\|T_{j, 2} f\right\|_{L \log L, \overline{\mathrm{Q}}} \\
& \leq C\|b\|_{\mathrm{BMO}} M_{L \log L, \varphi, \eta}\left(T_{j, 2} f\right)(x) .
\end{aligned}
$$

Write

$$
\begin{aligned}
& K_{j}^{\prime \prime} \\
& \leq \frac{1}{\varphi(|Q|)^{\rho_{2}}}\left(\frac{1}{|Q|} \int_{Q}\left|T_{j, 1} M_{\left(b-b_{\bar{Q}}\right) \chi_{(\bar{Q})}} T_{j, 2} f(y)\right| d y\right) .
\end{aligned}
$$

Let $L_{j}:=\left|T_{j, 1} M_{\left.\left(b-b_{\bar{Q}}\right) \chi_{(\bar{Q})}\right)^{c}} T_{j, 2} f(y)\right|, y \in Q$. Then, by taking $N=n \eta \alpha_{0}+1$ in Lemma 23, we have

$$
\begin{aligned}
L_{j} & \leq \int_{(\overline{\mathrm{Q}})^{c}}|K(y, z)|\left|b(z)-b_{\overline{\mathrm{Q}}}\right|\left|T_{j, 2} f(z)\right| d z \\
& \leq C \sum_{k=1}^{\infty} \int_{\mathrm{Q}_{k}} 2^{-k}\left|Q_{k}\right|^{-1} \varphi\left(\left|Q_{k}\right|\right)^{-\eta}\left|b(z)-b_{\overline{\mathrm{Q}}}\right| \\
& \cdot\left|T_{j, 2} f(z)\right| d z \leq C \sum_{k=1}^{\infty} 2^{-k}\left|Q_{k}\right|^{-1} \varphi\left(\left|Q_{k}\right|\right)^{-\eta} \\
& \cdot \int_{Q_{k}}\left|b(z)-b_{\mathrm{Q}_{k}}\right|\left|T_{j, 2} f(z)\right| d z
\end{aligned}
$$




$$
\begin{aligned}
& +C \sum_{k=1}^{\infty} 2^{-k}\left|Q_{k}\right|^{-1} \varphi\left(\left|Q_{k}\right|\right)^{-\eta} \int_{Q_{k}}\left|b_{Q_{k}}-b_{\bar{Q}}\right| \\
& \cdot\left|T_{j, 2} f(z)\right| d z:=L_{j}^{\prime}+L_{j}^{\prime \prime} .
\end{aligned}
$$

By the generalized Hölder inequality, we can get

$$
\begin{aligned}
L_{j}^{\prime} & \leq C \sum_{k=1}^{\infty} 2^{-k} \varphi\left(\left|Q_{k}\right|\right)^{-\eta}\|b\|_{\mathrm{BMO}}\left\|T_{j, 2} f\right\|_{L \log L, Q_{k}} \\
& \leq C\left(\sum_{k=1}^{\infty} 2^{-k}\right)\|b\|_{\mathrm{BMO}} M_{L \log L, \varphi, \eta}\left(T_{j, 2} f\right)(x) \\
& \leq C\|b\|_{\mathrm{BMO}} M_{L \log L, \varphi, \eta}\left(T_{j, 2} f\right)(x) .
\end{aligned}
$$

By Lemma 25, we have

$$
\begin{aligned}
& L_{j}^{\prime \prime} \leq C \sum_{k=1}^{\infty} 2^{-k}\left|Q_{k}\right|^{-1} \varphi\left(\left|Q_{k}\right|\right)^{-\eta} 2^{n} k\|b\|_{\mathrm{BMO}} \\
& \cdot \int_{Q_{k}}\left|T_{j, 2} f(z)\right| d z \leq C\left(\sum_{k=1}^{\infty} k 2^{-k}\right)\|b\|_{\mathrm{BMO}} \\
& \cdot M_{\varphi, \eta}\left(T_{j, 2} f\right)(x) \leq C\|b\|_{\mathrm{BMO}} M_{L \log L, \varphi, \eta}\left(T_{j, 2} f\right) \\
& \cdot(x) .
\end{aligned}
$$

Thus,

$$
L_{j} \leq C\|b\|_{\mathrm{BMO}} M_{L \log L, \varphi, \eta}\left(T_{j, 2} f\right)(x) .
$$

Then,

$$
K_{j}^{\prime \prime} \leq C\|b\|_{\mathrm{BMO}} M_{L \log L, \varphi, \eta}\left(T_{j, 2} f\right)(x) .
$$

Subcase 2. When $T_{j, 1}= \pm I$, take $1<q<\min \{\varepsilon / s, 1 /(1-s)\}$; then, $1<s q^{\prime}<\infty$ and $s q<\varepsilon$ :

$$
\begin{aligned}
K_{j}^{\prime} & =\frac{1}{\varphi(|Q|)^{\rho_{2}}}\left(\frac{1}{|Q|}\right. \\
& \left.\cdot \int_{Q}\left|b(y)-b_{\bar{Q}}\right|^{s}\left|T_{j, 2} f(y)\right|^{s} d y\right)^{1 / s} \\
& \leq \frac{1}{\varphi(|Q|)^{\rho_{2}}}\left(\frac{1}{|Q|} \int_{Q}\left|b(y)-b_{\bar{Q}}\right|^{s q^{\prime}} d y\right)^{1 /\left(s q^{\prime}\right)} \\
& \cdot\left(\frac{1}{|Q|} \int_{Q}\left|T_{j, 2} f(y)\right|^{s q} d y\right)^{1 /(s q)} \leq C\|b\|_{\mathrm{BMO}} \\
& \cdot \frac{1}{\varphi(|Q|)^{\rho_{2}}}\left(\frac{1}{|Q|} \int_{\mathrm{Q}}\left|T_{j, 2} f(y)\right|^{\varepsilon} d y\right)^{1 / \varepsilon} .
\end{aligned}
$$

Since $0<s<\varepsilon<1$, then $\varphi(|Q|)^{-\rho_{2}+\eta / \varepsilon} \leq 2^{\alpha_{0} \eta(1 / \varepsilon-1 / s)}$. Write

$$
\begin{aligned}
& \frac{1}{\varphi(|Q|)^{\rho_{2}}}\left(\frac{1}{|Q|} \int_{Q}\left|T_{j, 2} f(y)\right|^{\varepsilon} d y\right)^{1 / \varepsilon} \\
& =\varphi(|Q|)^{-\rho_{2}+\eta / \varepsilon}\left(\frac{1}{\varphi(|Q|)^{\eta}|Q|} \int_{Q}\left|T_{j, 2} f(y)\right|^{\varepsilon} d y\right)^{1 / \varepsilon} \\
& \leq C M_{\varepsilon, \varphi, \eta}^{\Delta}\left(T_{j, 2} f\right)(x) .
\end{aligned}
$$

Thus,

$$
\begin{aligned}
K_{j}^{\prime} & \leq C\|b\|_{\mathrm{BMO}} M_{\varepsilon, \varphi, \eta}^{\Delta}\left(T_{j, 2} f\right)(x) . \\
K_{j}^{\prime \prime} & =\frac{1}{\varphi(|Q|)^{\rho_{2}}}\left(\frac{1}{|\mathrm{Q}|}\right. \\
& \left.\cdot \int_{\mathrm{Q}}\left|\left(b(y)-b_{\overline{\mathrm{Q}}}\right) \chi_{(\overline{\mathrm{Q}})^{c}}(y) T_{j, 2} f(y)\right|^{s} d y\right)^{1 / s}=0 .
\end{aligned}
$$

So when $r \geq 1$,

$$
\begin{aligned}
& \sup _{\mathrm{Q} \ni x, r \geq 1}\left(\frac{1}{\varphi(|Q|)^{\eta}|Q|} \int_{Q}\left|T_{b} f(y)\right|^{s} d y\right)^{1 / s} \\
& \quad \leq C\|b\|_{\mathrm{BMO}} \\
& \quad \cdot \sum_{j=1}^{m}\left(M_{L \log L, \varphi, \eta}\left(T_{j, 2} f\right)(x)+M_{\varepsilon, \varphi, \eta}^{\Delta}\left(T_{j, 2} f\right)(x)\right) .
\end{aligned}
$$

Concluding the two cases, we can get

$$
\begin{aligned}
& M_{s, \varphi, \eta}^{\sharp, \Delta}\left(T_{b} f\right)(x) \leq C\|b\|_{\text {BMO }} \\
& \quad \cdot \sum_{j=1}^{m}\left(M_{L \log L, \varphi, \eta}\left(T_{j, 2} f\right)(x)+M_{\varepsilon, \varphi, \eta}^{\Delta}\left(T_{j, 2} f\right)(x)\right) .
\end{aligned}
$$

Lemma 28. Let $T \in L_{1, \delta}^{0}, 0<\delta<1, b \in B M O, 0<\eta<\infty$, and $0<s<\varepsilon<1$. Then, for any $f \in C_{0}^{\infty}\left(R^{n}\right)$,

$$
\begin{aligned}
& M_{s, \varphi, \eta}^{\sharp, \Delta}([b, T] f)(x) \\
& \quad \leq C\|b\|_{B M O}\left(M_{L \log L, \varphi, \eta} f(x)+M_{\varepsilon, \varphi, \eta}^{\Delta}(T f)(x)\right) .
\end{aligned}
$$

The proof is similar to that of Lemma 27; thus, we omit the details here.

Lemma 29 (see [12]). Let $\omega \in A_{1}(\varphi), \eta \geq 2$, and $B(t)=t(1+$ $\left.\log ^{+} t\right)$. Then, there exists a constant $C>0$ such that, for any function $f$ and $\lambda>0$,

$$
\begin{gathered}
\omega\left(\left\{x \in R^{n}: M_{L \log L, \varphi, \eta}(f)(x)>\lambda\right\}\right) \\
\leq C \int_{R^{n}} B\left(\frac{|f(y)|}{\lambda}\right) \omega(y) d y .
\end{gathered}
$$


Lemma 30. Let $T \in L_{1, \delta}^{0}, 0<\delta<1, b \in B M O, 0<\eta<$ $\infty, \omega \in A_{1}(\varphi)$, and $B(t)=t\left(1+\log ^{+} t\right)$. Then, there exists a constant $C>0$ such that, for any $f \in C_{0}^{\infty}\left(R^{n}\right)$,

$$
\begin{aligned}
\sup _{t>0} & \frac{1}{B(1 / t)} \omega\left(\left\{x \in R^{n}:|[b, T] f(x)|>t\right\}\right) \\
& \leq C\|b\|_{B M O} \\
& \cdot \sup _{t>0} \frac{1}{B(1 / t)} \omega\left(\left\{x \in R^{n}: M_{L \log L, \varphi, \eta} f(x)>t\right\}\right) .
\end{aligned}
$$

Proof. Taking $0<s<\varepsilon<1$, by Lebesgue differential theorem, we have a.e. for $x \in R^{n}$

$$
|[b, T] f(x)| \leq M_{s, \varphi, \eta}^{\Delta}([b, T] f)(x) .
$$

Thus, we get

$$
\begin{aligned}
& \sup _{t>0} \frac{1}{B(1 / t)} \omega\left(\left\{x \in R^{n}:|[b, T] f(x)|>t\right\}\right) \\
& \quad \leq \sup _{t>0} \frac{1}{B(1 / t)} \\
& \cdot \omega\left(\left\{x \in R^{n}: M_{s, \varphi, \eta}^{\Delta}([b, T] f)(x)>t\right\}\right) .
\end{aligned}
$$

Then, we only need to show that

$$
\begin{aligned}
& \sup _{t>0} \frac{1}{B(1 / t)} \omega\left(\left\{x \in R^{n}: M_{s, \varphi, \eta}^{\Delta}([b, T] f)(x)>t\right\}\right) \\
& \leq C\|b\|_{\mathrm{BMO}} \sup _{t>0} \frac{1}{B(1 / t)} \\
& \cdot \omega\left(\left\{x \in R^{n}: M_{L \log L, \varphi, \eta} f(x)>t\right\}\right) .
\end{aligned}
$$

Let

$$
\begin{aligned}
& L_{s}(f):=\sup _{t>0} \frac{1}{B(1 / t)} \\
& \cdot \omega\left(\left\{x \in R^{n}: M_{s, \varphi, \eta}^{\Delta}([b, T] f)(x)>t\right\}\right) .
\end{aligned}
$$

By Lemma 21, for any $t>0$ and $0<\gamma<b<b_{0}=1 / \varphi\left(2^{n}\right)^{\eta}$, there is

$$
\begin{aligned}
& \omega\left(\left\{x \in R^{n}: M_{s, \varphi, \eta}^{\Delta}([b, T] f)(x)>t\right\}\right)=\omega(\{x \\
& \left.\left.\quad \in R^{n}: M_{\varphi, \eta}^{\Delta}\left(|[b, T] f|^{s}\right)(x)>t^{s}\right\}\right) \leq \omega(\{x \\
& \quad \in R^{n}: M_{\varphi, \eta}^{\Delta}\left(|[b, T] f|^{s}\right)(x) \\
& \left.\left.\quad>t^{s}, M_{\varphi, \eta}^{\sharp, \Delta}\left(|[b, T] f|^{s}\right)(x) \leq \gamma t^{s}\right\}\right)+\omega(\{x \\
& \quad \in R^{n}: M_{\varphi, \eta}^{\Delta}\left(|[b, T] f|^{s}\right)(x) \\
& \left.\left.>t^{s}, M_{\varphi, \eta}^{\sharp, \Delta}\left(|[b, T] f|^{s}\right)(x)>\gamma t^{s}\right\}\right) \leq C a^{\delta_{1}} \omega(\{x \\
& \left.\left.\quad \in R^{n}: M_{\varphi, \eta}^{\Delta}\left(|[b, T] f|^{s}\right)(x)>b t^{s}\right\}\right)+\omega(\{x \\
& \left.\left.\quad \in R^{n}: M_{\varphi, \eta}^{\sharp, \Delta}\left(|[b, T] f|^{s}\right)(x)>\gamma t^{s}\right\}\right):=M+N .
\end{aligned}
$$

Let $d=\gamma^{1 / s} / 2 C\|b\|_{\text {BMO }}$; by Lemma 28 , we have

$$
\begin{aligned}
N & =\omega\left(\left\{x \in R^{n}: M_{s, \varphi, \eta}^{\sharp, \Delta}([b, T] f)(x)>\gamma^{1 / s} t\right\}\right) \\
& \leq \omega\left(\left\{x \in R^{n}: M_{L \log L, \varphi, \eta} f(x)+M_{\varepsilon, \varphi, \eta}^{\Delta}(T f)(x)\right.\right. \\
& \left.\left.>\frac{\gamma^{1 / s} t}{C\|b\|_{\mathrm{BMO}}}\right\}\right) \leq \omega\left(\left\{x \in R^{n}: M_{L \log L, \varphi, \eta} f(x)\right.\right. \\
& \left.\left.>\frac{\gamma^{1 / s} t}{2 C\|b\|_{\mathrm{BMO}}}\right\}\right)+\omega\left(\left\{x \in R^{n}: M_{\varepsilon, \varphi, \eta}^{\Delta}(T f)(x)\right.\right. \\
& \left.\left.>\frac{\gamma^{1 / s} t}{2 C\|b\|_{\mathrm{BMO}}}\right\}\right)=\omega\left(\left\{x \in R^{n}: M_{L \log L, \varphi, \eta} f(x)\right.\right. \\
& >d t\})+\omega\left(\left\{x \in R^{n}: M_{\varepsilon, \varphi, \eta}^{\Delta}(T f)(x)>d t\right\}\right) .
\end{aligned}
$$

Thus, we can obtain

$$
\begin{aligned}
\omega(\{x & \left.\left.\in R^{n}: M_{s, \varphi, \eta}^{\Delta}([b, T] f)(x)>t\right\}\right) \\
\leq & C a^{\delta_{1}} \omega\left(\left\{x \in R^{n}: M_{s, \varphi, \eta}^{\Delta}([b, T] f)(x)>b^{1 / s} t\right\}\right) \\
& +\omega\left(\left\{x \in R^{n}: M_{L \log L, \varphi, \eta} f(x)>d t\right\}\right) \\
& +\omega\left(\left\{x \in R^{n}: M_{\varepsilon, \varphi, \eta}^{\Delta}(T f)(x)>d t\right\}\right) .
\end{aligned}
$$

Let $b^{1 / s} t=t^{\prime}$; then, $1 / t=b^{1 / s} / t^{\prime}$. Let $d t=t^{\prime \prime}$; then, $1 / t=$ $d / t^{\prime \prime}$. Since, for any $a \geq 0$ and $b \geq 0, B(a b) \leq B(a) B(b)$, then $B\left(b^{1 / s} / t^{\prime}\right) \geq C_{b} B\left(1 / t^{\prime}\right)$ and $B\left(d / t^{\prime \prime}\right) \geq C_{d} B\left(1 / t^{\prime \prime}\right)$. Dividing by $B(1 / t)$ in both sides of the above inequality, we get

$$
\begin{aligned}
& \frac{1}{B(1 / t)} \omega\left(\left\{x \in R^{n}: M_{s, \varphi, \eta}^{\Delta}([b, T] f)(x)>t\right\}\right) \\
& \leq \frac{C a^{\delta_{1}}}{B\left(b^{1 / s} / t^{\prime}\right)} \\
& \cdot \omega\left(\left\{x \in R^{n}: M_{s, \varphi, \eta}^{\Delta}([b, T] f)(x)>t^{\prime}\right\}\right) \\
& +\frac{1}{B\left(d / t^{\prime \prime}\right)} \omega\left(\left\{x \in R^{n}: M_{L \log L, \varphi, \eta} f(x)>t^{\prime \prime}\right\}\right) \\
& +\frac{1}{B\left(d / t^{\prime \prime}\right)} \omega\left(\left\{x \in R^{n}: M_{\varepsilon, \varphi, \eta}^{\Delta}(T f)(x)>t^{\prime \prime}\right\}\right) \\
& \leq \frac{C_{b} a^{\delta_{1}}}{B\left(1 / t^{\prime}\right)} \\
& \cdot \omega\left(\left\{x \in R^{n}: M_{s, \varphi, \eta}^{\Delta}([b, T] f)(x)>t^{\prime}\right\}\right) \\
& +\frac{C_{d}}{B\left(1 / t^{\prime \prime}\right)} \omega\left(\left\{x \in R^{n}: M_{L \log L, \varphi, \eta} f(x)>t^{\prime \prime}\right\}\right) \\
& +\frac{C_{d}}{B\left(1 / t^{\prime \prime}\right)} \omega\left(\left\{x \in R^{n}: M_{\varepsilon, \varphi, \eta}^{\Delta}(T f)(x)>t^{\prime \prime}\right\}\right) .
\end{aligned}
$$


Then, we can achieve that

$$
\begin{aligned}
& \frac{1}{B(1 / t)} \omega\left(\left\{x \in R^{n}: M_{s, \varphi, \eta}^{\Delta}([b, T] f)(x)>t\right\}\right) \\
& \leq \sup _{t>0} \frac{C a^{\delta_{1}}}{B(1 / t)} \\
& \cdot \omega\left(\left\{x \in R^{n}: M_{s, \varphi, \eta}^{\Delta}([b, T] f)(x)>t\right\}\right) \\
& +\sup _{t>0} \frac{C\|b\|_{\mathrm{BMO}}}{B(1 / t)} \\
& \cdot \omega\left(\left\{x \in R^{n}: M_{L \log L, \varphi, \eta} f(x)>t\right\}\right) \\
& +\sup _{t>0} \frac{C\|b\|_{\mathrm{BMO}}}{B(1 / t)} \\
& \cdot \omega\left(\left\{x \in R^{n}: M_{\varepsilon, \varphi, \eta}^{\Delta}(T f)(x)>t\right\}\right) .
\end{aligned}
$$

Then,

$$
\begin{aligned}
L_{s}(f) \leq & C a^{\delta_{1}} L_{s}(f)+C\|b\|_{\mathrm{BMO}} \sup _{t>0} \frac{1}{B(1 / t)} \\
& \cdot \omega\left(\left\{x \in R^{n}: M_{L \log L, \varphi, \eta} f(x)>t\right\}\right) \\
+ & C\|b\|_{\mathrm{BMO}} \sup _{t>0} \frac{1}{B(1 / t)} \\
& \cdot \omega\left(\left\{x \in R^{n}: M_{\varepsilon, \varphi, \eta}^{\Delta}(T f)(x)>t\right\}\right) .
\end{aligned}
$$

By Lemmas $16,18(\mathrm{a})$, and 25 , denoting $t^{\prime \prime \prime}=t / C$, we have

$$
\begin{aligned}
& C\|b\|_{\mathrm{BMO}} \sup _{t>0} \frac{1}{B(1 / t)} \\
& \cdot \omega\left(\left\{x \in R^{n}: M_{\varepsilon, \varphi, \eta}^{\Delta}(T f)(x)>t\right\}\right) \leq C\|b\|_{\mathrm{BMO}} \\
& \cdot \sup _{t>0} \frac{1}{B(1 / t)} \omega\left(\left\{x \in R^{n}: M_{\varepsilon, \varphi, \eta}^{\sharp, \Delta}(T f)(x)>t\right\}\right) \\
& \leq C\|b\|_{\mathrm{BMO}} \sup _{t>0} \frac{1}{B(1 / t)} \\
& \cdot \omega\left(\left\{x \in R^{n}: M_{\varphi, \eta} f(x)>\frac{t}{C}\right\}\right)=C\|b\|_{\mathrm{BMO}} \\
& \cdot \sup _{t>0} \frac{1}{B\left(1 /\left(C t^{\prime \prime \prime}\right)\right)} \omega\left(\left\{x \in R^{n}: M_{\varphi, \eta} f(x)>t^{\prime \prime \prime}\right\}\right) \\
& \leq C\|b\|_{\mathrm{BMO}} \sup _{t^{\prime \prime \prime}>0} \frac{1}{B\left(1 / t^{\prime \prime \prime}\right)} \\
& \cdot \omega\left(\left\{x \in R^{n}: M_{\varphi, \eta} f(x)>t^{\prime \prime \prime}\right\}\right) \leq C\|b\|_{\mathrm{BMO}} \\
& \cdot \sup _{t^{\prime \prime \prime}>0} \frac{1}{B\left(1 / t^{\prime \prime \prime}\right)} \\
& \cdot \omega\left(\left\{x \in R^{n}: M_{L \log L, \varphi, \eta} f(x)>\frac{t^{\prime \prime \prime}}{C}\right\}\right)
\end{aligned}
$$

$$
\begin{aligned}
& \leq C\|b\|_{\mathrm{BMO}} \sup _{t^{\prime \prime \prime}>0} \frac{1}{B\left(C / t^{\prime \prime \prime}\right)} \\
& \cdot \omega\left(\left\{x \in R^{n}: M_{L \log L, \varphi, \eta} f(x)>\frac{t^{\prime \prime \prime}}{C}\right\}\right) \\
& =C\|b\|_{\mathrm{BMO}} \sup _{t>0} \frac{1}{B(1 / t)} \\
& \cdot \omega\left(\left\{x \in R^{n}: M_{L \log L, \varphi, \eta} f(x)>t\right\}\right) .
\end{aligned}
$$

It follows from Lemma 21 that $a=2^{n} \gamma /\left(1-b / b_{0}\right)$. Take $\gamma$ such that $1-C a^{\delta_{1}}>0$; then,

$$
\begin{aligned}
L_{s}(f) \leq & C\|b\|_{\mathrm{BMO}} \sup _{t>0} \frac{1}{B(1 / t)} \\
& \cdot \omega\left(\left\{x \in R^{n}: M_{L \log L, \varphi, \eta} f(x)>t\right\}\right) .
\end{aligned}
$$

From the above, the following inequality holds:

$$
\begin{gathered}
\sup _{t>0} \frac{1}{B(1 / t)} \omega\left(\left\{x \in R^{n}:|[b, T] f(x)|>t\right\}\right) \\
\leq C\|b\|_{\mathrm{BMO}} \sup _{t>0} \frac{1}{B(1 / t)} \\
\cdot \omega\left(\left\{x \in R^{n}: M_{L \log L, \varphi, \eta} f(x)>t\right\}\right) .
\end{gathered}
$$

\section{Proof of Main Results}

Now we are able to prove our main results.

Proof of Theorem 8. Take $p^{\prime}<\eta<\infty$; then, for all $x \in R^{n}$,

$$
M_{\varphi, \eta} f(x) \leq M_{\varphi, p^{\prime}} f(x) .
$$

Taking $0<s<1$, by Lemmas $16-18$, we get

$$
\begin{aligned}
\|T f\|_{L^{p}(\omega)} & \leq C\left\|M_{s, \varphi, \eta}^{\sharp, \Delta}(T f)\right\|_{L^{p}(\omega)} \leq C\left\|M_{\varphi, \eta} f\right\|_{L^{p}(\omega)} \\
& \leq C\left\|M_{\varphi, p^{\prime}} f\right\|_{L^{p}(\omega)} \leq C\|f\|_{L^{p}(\omega)} .
\end{aligned}
$$

Proof of Theorem 9. For any $0<s<1$ and $1 \leq \eta<\infty$, we have

$$
\begin{array}{r}
|T f(x)| \leq\left(M_{\varphi, \eta}^{\Delta}\left(|T f|^{s}\right)(x)\right)^{1 / s}=M_{s, \varphi, \eta}^{\Delta}(T f)(x) \\
\text { a.e. } x \in R^{n}, \\
M_{\varphi, \eta} f(x) \leq M_{\varphi} f(x) .
\end{array}
$$

Thus,

$$
\begin{aligned}
& \left\{x \in R^{n}: M_{\varphi, \eta} f(x)>\lambda\right\} \\
& \quad \subset\left\{x \in R^{n}: M_{\varphi} f(x)>\lambda\right\} .
\end{aligned}
$$


Taking $\psi(\lambda)=\lambda$ in Lemma 18 and applying Lemmas 13 and 16 , we can get

$$
\begin{aligned}
& \sup _{\lambda>0} \lambda \cdot \omega\left(\left\{y \in R^{n}:|T f(y)|>\lambda\right\}\right) \\
& \leq \sup _{\lambda>0} \lambda \cdot \omega\left(\left\{y \in R^{n}: M_{s, \varphi, \eta}^{\Delta}(T f)(y)>\lambda\right\}\right) \\
& \leq C \sup _{\lambda>0} \lambda \cdot \omega\left(\left\{y \in R^{n}: M_{s, \varphi, \eta}^{\sharp, \Delta}(T f)(y)>\lambda\right\}\right) \\
& \leq C \sup _{\lambda>0} \lambda \cdot \omega\left(\left\{y \in R^{n}: C M_{\varphi, \eta} f(y)>\lambda\right\}\right) \\
& \leq C \sup _{\lambda>0} \lambda \cdot \omega\left(\left\{y \in R^{n}: M_{\varphi} f(y)>\frac{\lambda}{C}\right\}\right) \\
& \leq C \int_{R^{n}}|f(y)| \omega(y) d y .
\end{aligned}
$$

Thus, for any $\lambda>0$, the desired inequality holds.

Proof of Theorem 10. Take $\eta=2 p^{\prime}$ and $0<s<\varepsilon<1$. Then, $\varphi(|Q|)^{p^{\prime}}<\varphi(|Q|)^{\eta}$, and

$$
\left\|M_{\varphi, \eta}(f)\right\|_{L^{p}(\omega)} \leq\left\|M_{\varphi, p^{\prime}} f\right\|_{L^{p}(\omega)} .
$$

It follows from Lemmas 16-18, 20, and 26-27 and Theorem 8 that

$$
\begin{aligned}
& \left\|T_{b}(f)\right\|_{L^{p}(\omega)} \leq C\left\|M_{s, \varphi, \eta}^{\sharp, \Delta}\left(T_{b} f\right)\right\|_{L^{p}(\omega)} \\
& \leq C\|b\|_{\mathrm{BMO}} \sum_{j=1}^{m}\left\|M_{L \log L, \varphi, \eta}\left(T_{j, 2} f\right)\right\|_{L^{p}(\omega)} \\
& +C\|b\|_{\text {BMO }} \sum_{j=1}^{m}\left\|M_{\varepsilon, \varphi, \eta}^{\Delta}\left(T_{j, 2} f\right)\right\|_{L^{p}(\omega)} \\
& \leq C\|b\|_{\mathrm{BMO}} \sum_{j=1}^{m}\left\|M_{\varphi, \eta / 2} M_{\varphi, \eta / 2}\left(T_{j, 2} f\right)\right\|_{L^{p}(\omega)} \\
& +C\|b\|_{\mathrm{BMO}} \sum_{j=1}^{m}\left\|M_{\varphi, \eta} f\right\|_{L^{p}(\omega)} \\
& \leq C\|b\|_{\mathrm{BMO}} \sum_{j=1}^{m}\left\|M_{\varphi, p^{\prime}} M_{\varphi, p^{\prime}}\left(T_{j, 2} f\right)\right\|_{L^{p}(\omega)} \\
& +C\|b\|_{\mathrm{BMO}} \sum_{j=1}^{m}\left\|M_{\varphi, p^{\prime}} f\right\|_{L^{p}(\omega)} \\
& \leq C\|b\|_{\mathrm{BMO}} \sum_{j=1}^{m}\left\|T_{j, 2} f\right\|_{L^{p}(\omega)} \\
& +C\|b\|_{\mathrm{BMO}} \sum_{j=1}^{m}\|f\|_{L^{p}(\omega)} \\
& \leq C\|b\|_{\mathrm{BMO}} \sum_{j=1}^{m}\left\|T_{j, 2}\right\|\|f\|_{L^{p}(\omega)}
\end{aligned}
$$

$$
\begin{gathered}
+C\|b\|_{\mathrm{BMO}}\|f\|_{L^{p}(\omega)} \\
\leq C\|b\|_{\mathrm{BMO}}\left(\sum_{j=1}^{m}\left\|T_{j, 2}\right\|+1\right)\|f\|_{L^{p}(\omega)} .
\end{gathered}
$$

Thus, $T_{b}$ is bounded on $L^{p}(\omega)$.

Proof of Theorem 12. Let $B(t)=t\left(1+\log ^{+} t\right)$. By homogeneity, we need only to show that

$$
\begin{gathered}
\omega\left(\left\{x \in R^{n}:|[b, T] f(x)|>1\right\}\right) \\
\quad \leq C \int_{R^{n}} B(|f(x)|) \omega(x) d x .
\end{gathered}
$$

Taking $\eta \geq 2$, by Lemma 29-30, we get

$$
\begin{aligned}
& \omega\left(\left\{x \in R^{n}:|[b, T] f(x)|>1\right\}\right) \leq C \sup _{t>0} \frac{1}{B(1 / t)} \\
& \cdot \omega\left(\left\{x \in R^{n}:|[b, T] f(x)|>t\right\}\right) \leq C\|b\|_{\mathrm{BMO}} \\
& \cdot \sup _{t>0} \frac{1}{B(1 / t)} \omega\left(\left\{x \in R^{n}: M_{L \log L, \varphi, \eta} f(x)>t\right\}\right) \\
& \leq C\|b\|_{\mathrm{BMO}} \sup _{t>0} \frac{1}{B(1 / t)} \int_{R^{n}} B\left(\frac{|f(x)|}{t}\right) \omega(x) d x \\
& \leq C\|b\|_{\mathrm{BMO}} \sup _{t>0} \frac{1}{B(1 / t)} \\
& \cdot \int_{R^{n}} B(|f(x)|) B\left(\frac{1}{t}\right) \omega(x) d x \leq C\|b\|_{\mathrm{BMO}} \\
& \cdot \int_{R^{n}} B(|f(x)|) \omega(x) d x .
\end{aligned}
$$

Thus, the desired result holds.

\section{Competing Interests}

The authors declare that there is no conflict of interests regarding the publication of this paper.

\section{Acknowledgments}

This work was supported by the National Natural Science Foundation of China (11171345), Beijing Higher Education Young Elite Teacher Project (YETP0946), the Fundamental Research Funds for the Central Universities (2009QS16), and the State Scholarship Fund of China.

\section{References}

[1] J. J. Kohn and L. Nirenberg, "An algebra of pseudo-differential operators," Communications on Pure and Applied Mathematics, vol. 18, pp. 269-305, 1965.

[2] L. Hörmander, "Pseudo-differential operators," Communications on Pure and Applied Mathematics, vol. 18, pp. 501-517, 1965. 
[3] L. Hörmander, "Pseudo-differential operators and hypoelliptic equations," Proceeding of Symposia in Pure Mathematics, vol. 10, pp. 138-183, 1967.

[4] A. A. Laptev, "Spectral asymptotics of a class of Fourier integral operators," Trudy Moskovskogo Mathematics Obshchestva, vol. 43, pp. 92-115, 1981 (Russian).

[5] C. Fefferman, " $L p$-bounds for a class of pseudo-differential operators," Israel Journal of Mathematics, vol. 14, pp. 413-417, 1973.

[6] J. Alvarez and J. Hounie, "Estimates for the kernel and continuity properties of pseudo-differential operators," Arkiv för Matematik, vol. 28, no. 1, pp. 1-22, 1990.

[7] S. Chanillo, "Remarks on commutators of pseudo-differential operators," Contemporary Mathematics, vol. 205, pp. 33-37, 1997.

[8] S. Chanillo and A. Torchinsky, "Sharp function and weighted $L_{p}$ estimates for a class of pseudo-differential operators," Arkiv för Matematik, vol. 24, pp. 1-25, 1986.

[9] Y. Lin and S. Z. Lu, "Pseudo-differential operators on Sobolev and Lipschitz spaces," Acta Mathematica Sinica, vol. 26, no. 1, pp. 131-142, 2010.

[10] E. M. Stein, Singular Integrals and Differentiability Properties of Functions, Princeton Mathematical Series no. 30, Princeton University Press, Princeton, NJ, USA, 1970.

[11] N. Miller, "Weighted sobolev spaces and pseudo-differential operators with smooth symbols," Transactions of the American Mathematical Society, vol. 269, no. 1, pp. 91-109, 1982.

[12] L. Tang, "Weighted norm inequalities for pseudo-differential operators with smooth symbols and their commutators," Journal of Functional Analysis, vol. 262, no. 4, pp. 1603-1629, 2012.

[13] J. Garcia-Cuerva and J. R. de Francia, Weighted Norm Inequalities and Related Topics, North-Holland, Amsterdam, The Netherlands, 1985.

[14] R. Coifman, R. Rochberg, and G. Weiss, "Factorization theorems for Hardy spaces in several variables," Journal of Functional Analysis, vol. 103, pp. 611-635, 1976.

[15] P. Auscher and M. E. Taylor, "Paradifferential operators and commutator estimates," Communications in Partial Differential Equations, vol. 20, no. 9-10, pp. 1743-1775, 1995.

[16] M. Taylor, "Commutator estimates," Proceedings of the American Mathematical Society, vol. 131, no. 5, pp. 1501-1507, 2003.

[17] Y. Lin, "Commutators of pseudo-differential operators," Science in China Series A: Mathematics, vol. 51, no. 3, pp. 453-460, 2008.

[18] N. Michalowski, D. J. Rule, and W. Staubach, "Weighted norm inequalities for pseudo-pseudodifferential operators defined by amplitudes," Journal of Functional Analysis, vol. 258, no. 12, pp. 4183-4209, 2010.

[19] S. G. Krantz and S. Y. Li, "Boundedness and compactness of integral operators on spaces of homogeneous type and applications," Journal of Mathematical Analysis and Applications, vol. 258, no. 2, pp. 629-641, 2001

[20] R. Coifman, "A real variable characterization of $H^{p}$," Studia Mathematica, vol. 51, pp. 269-274, 1974.

[21] C. Fefferman and E. M. Stein, " $H^{p}$ spaces of several variables," Acta Mathematica, vol. 129, no. 3-4, pp. 137-193, 1972.

[22] M. Paluszyński, "Characterization of the Besov spaces via the commutator operator of Coifman, Rochberg and Weiss," Indiana University Mathematics Journal, vol. 44, no. 1, pp. 1-17, 1995.

[23] C. Pérez, "Endpoint estimates for commutators of singular integral operators," Journal of Functional Analysis, vol. 128, no. 1, pp. 163-185, 1995.
[24] E. M. Stein, Harmonic Analysis: Real-Variable Methods, Orthogonality, and Oscillatory Integrals, vol. 43 of Princeton Mathematical Series, Princeton University Press, Princeton, NJ, USA, 1993. 


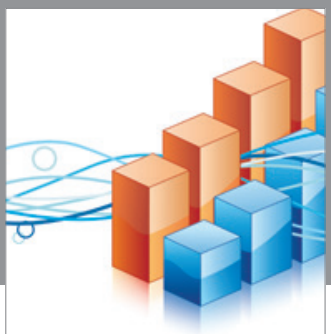

Advances in

Operations Research

vatem alat4

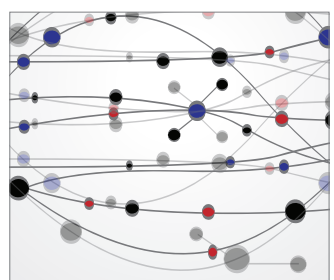

\section{The Scientific} World Journal
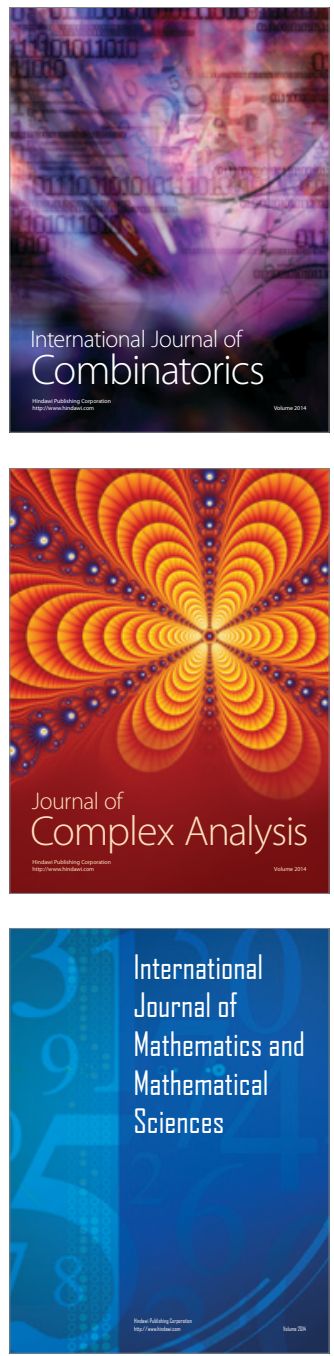
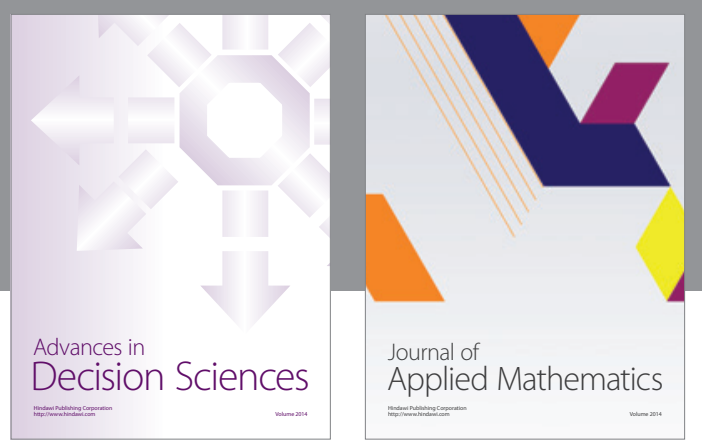

Algebra

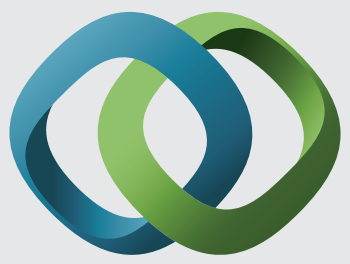

\section{Hindawi}

Submit your manuscripts at

http://www.hindawi.com
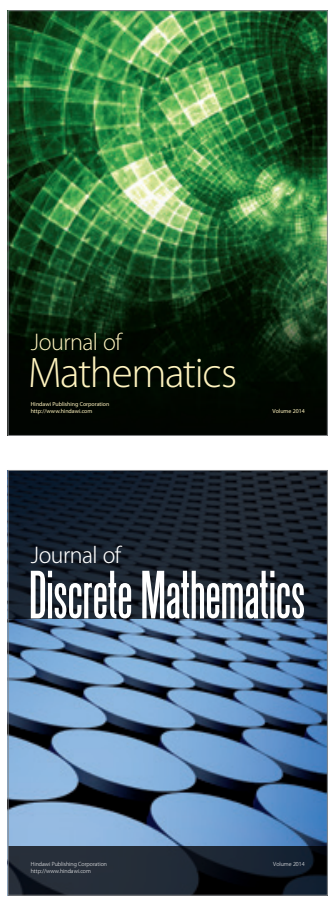

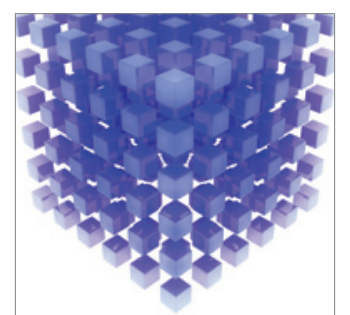

Mathematical Problems in Engineering
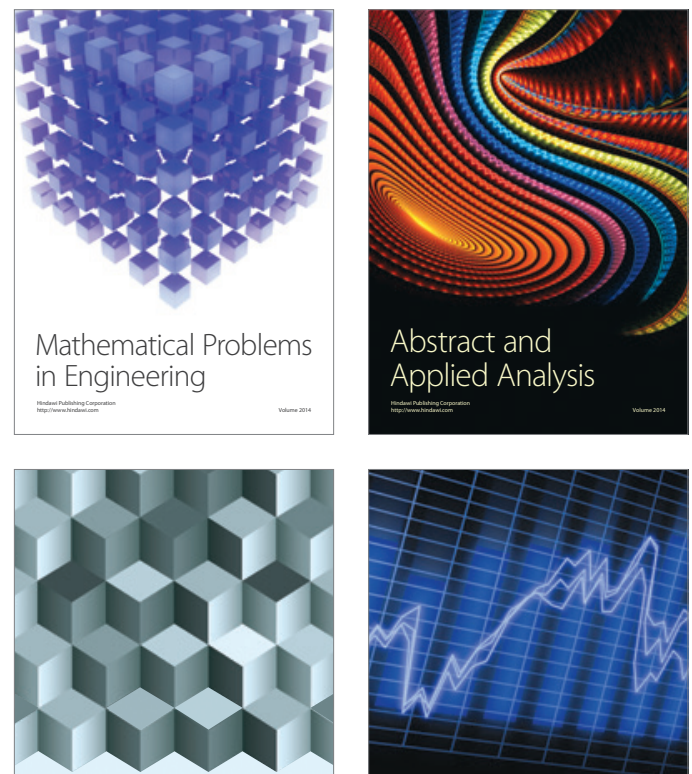

Journal of

Function Spaces

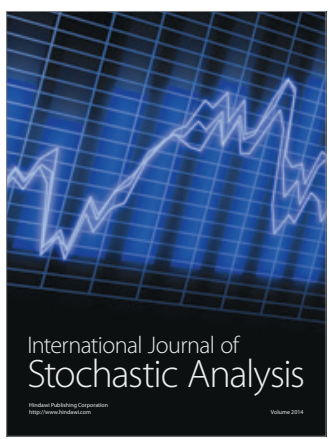

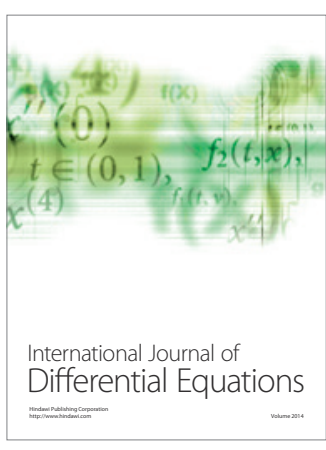
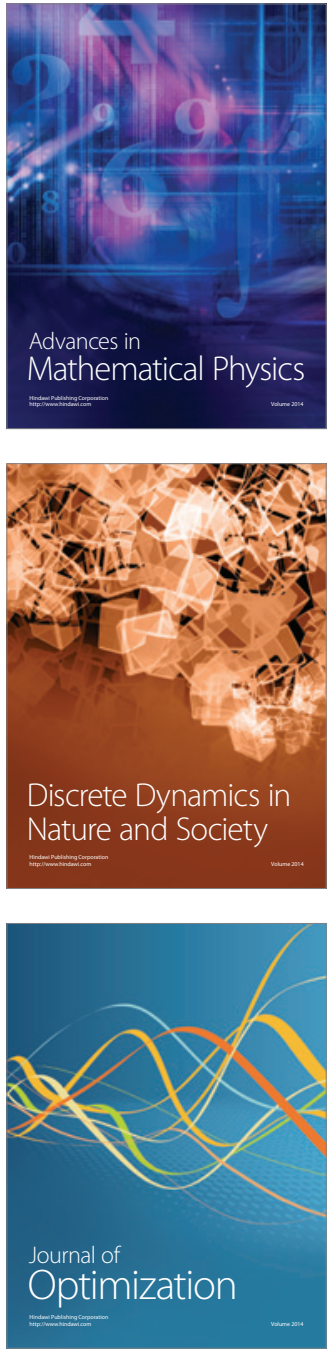